\title{
POISSON project
}

\section{Emission lines as accretion tracers in young stellar objects: results from observations of Chamaeleon I and II sources ${ }^{\star}$}

\author{
S. Antoniucci ${ }^{1}$, R. García López ${ }^{1,2}$, B. Nisini ${ }^{1}$, T. Giannini ${ }^{1}$, D. Lorenzetti ${ }^{1}$, J. Eislöffel ${ }^{3}$, \\ F. Bacciotti ${ }^{4}$, S. Cabrit ${ }^{5}$, A. Caratti o Garatti ${ }^{6}$, C. Dougados ${ }^{7}$, and T. Ray ${ }^{6}$
}

\author{
${ }^{1}$ INAF-Osservatorio Astronomico di Roma, via di Frascati 33, 00040 Monte Porzio Catone, Italy \\ e-mail: simone.antoniucci@oa-roma.astro.it \\ 2 Max-Planck-Institut für Radioastronomie, Auf dem Hügel 69, 53121 Bonn, Germany \\ 3 Thüringer Landessternwarte Tautenburg, Sternwarte 5, 07778 Tautenburg, Germany \\ 4 INAF-Osservatorio Astrofisico di Arcetri, Largo Enrico Fermi 5, 50125 Firenze, Italy \\ ${ }^{5}$ Laboratoire d'Études du Rayonnement et de la Matière en Astrophysique (LERMA), Observatoire de Paris, ENS, UPMC, UCP, \\ CNRS, 61 Avenue de l'Observatoire, 75014 Paris, France \\ ${ }_{7}^{6}$ Dublin Institute for Advanced Studies, School of Cosmic Physics, 31 Fitzwilliam Place, Dublin 2, Ireland \\ 7 Institut de Planetologie et d'Astrophysique de Grenoble (IPAG), UMR 5571, BP 53, 38041 Grenoble Cedex 09, France
}

Received 9 June 2011 / Accepted 11 August 2011

\begin{abstract}
Context. We present the results of the analysis of low-resolution optical-near IR spectroscopy (0.6-2.4 $\mu \mathrm{m})$ of a sample (47 sources) of Class I and Class II young stellar objects in the Chamaeleon I and II star-forming clouds. These data are part of the POISSON project (Protostellar Optical-Infrared Spectral Survey On NTT).

Aims. The aim of the observations is to determine the accretion luminosity $\left(L_{\text {acc }}\right)$ and mass accretion rate $\left(\dot{M}_{\text {acc }}\right)$ of the sources through the analysis of the detected emission features. Taking advantage of the wide wavelength range covered by our spectra, we also aim at verifying the reliability and consistency of the existing empirical relationships connecting emission line luminosity and $L_{\text {acc }}$.

Methods. We employ five different tracers ([O I $] \lambda 6300, \mathrm{H} \alpha, \mathrm{Ca}$ II $\lambda 8542, \mathrm{~Pa} \beta$, and $\mathrm{Br} \gamma$ ) to derive the accretion luminosity, and critically discuss the various determinations in the light of the source properties.

Results. The tracers provide $L_{\text {acc }}$ values characterised by different scatters when plotted as a function of $L_{*}$. The $\operatorname{Br} \gamma$ relation appears to be the most reliable, because it gives the minimum dispersion of $L_{\text {acc }}$ over the entire range of $L_{*}$, whereas the other tracers, in particular $\mathrm{H} \alpha$, provide much more scattered $L_{\mathrm{acc}}$ results, which are not expected for the homogeneous sample of targets we are observing. The direct comparison between $L_{\mathrm{acc}}(\mathrm{Br} \gamma)$ and the accretion luminosity obtained from the other four tracers also shows systematic differences in the results provided by the empirical relationships. These may probably be ascribed to different excitation mechanisms that contribute to the line emission, which may vary between our sample and those where the relationships have been calibrated, which were mostly based on observations in Taurus. Adopting the accretion luminosities estimates derived from the Br $\gamma$ line, we infer $L_{\text {acc }}$ in the range $0.1 L_{*}-1 L_{*}$ for all sources, and $\dot{M}_{\text {acc }}$ of the order $10^{-7}-10^{-9} M_{\odot} \mathrm{yr}^{-1}$, in the range of values commonly obtained for Class II objects. The mass accretion rates derived in Cha I are roughly proportional to $M_{*}^{2}$, in agreement with the results found in other low-mass star-forming regions. We find that the discrepancies observed in the case of $L_{\text {acc }}(\operatorname{Br} \gamma)$ and $L_{\text {acc }}(\operatorname{Pa} \beta)$ can be related to different intrinsic $\mathrm{Pa} \beta / \mathrm{Br} \gamma$ ratios. The derived ratios point to the existence of two different emission modalities, one that agrees with predictions of both wind and accretion models, the other suggesting optically thick emission from relatively small regions $\left(10^{21}-10^{22} \mathrm{~cm}^{2}\right)$ with gas at low temperatures $(<4000 \mathrm{~K})$, the origin of which needs additional investigation.
\end{abstract}

Key words. stars: evolution - techniques: spectroscopic - accretion, accretion disks - stars: formation - surveys

\section{Introduction}

One of the major observational challenges in star-formation studies is the derivation of the relevant physical parameters characterising the accretion process in young stellar objects (YSOs) and the determination of their evolutionary status.

Traditionally, YSOs are divided into evolutionary classes that are empirically based on the shape of their spectral energy distribution (SED) (Class 0, I, II, and III objects, Lada \& Wilking 1984; Andre et al. 1993). This classification reflects the evolution of the source circumstellar material, from a massive

^ Based on observations collected at the European Southern Observatory, La Silla, Chile (ESO programme 082.C-0264). and thick envelope in Class 0 to a tenuous circumstellar disc in Class III, but it does not give direct information on the accretion activity of the objects, nor on how this is related to the source/disc system and how it evolves with time. There is indeed evidence that only a fraction of Class I sources have mass accretion rates high enough to put them in their main stage of mass-building (Enoch et al. 2009; Evans et al. 2009; Antoniucci et al. 2008; Beck 2007; Nisini et al. 2005a; White \& Hillenbrand 2004), while there is a wide difference in disc activity among Classical T Tauri Stars (CTTSs, Class II objects) of the same mass (e.g. Gullbring et al. 1998). This evidence indicates that the standard scenario in which most of the object mass is accumulated during the Class 0/I phase has to be revised: possible 
alternative pictures are that either the stellar mass is assembled within the Class 0 stage, or YSOs undergo periods of eruptive events of enhanced mass accretion. To distinguish the possible interpretations, it is necessary to study large samples of sources of different classes and to compare their mass and age with their accretion properties.

From the observational point of view, important quantitative information on the active inner and complex region of interaction between the accretion disc, the young star, and the jet/wind formation region can be obtained with observations of the many diagnostic emission/absorption lines available in the optical/near-IR wavelength range. Several spectroscopic studies have been carried out on samples of young objects with the aim of deriving their main stellar parameters and of inferring relevant disc parameters. In particular, the mass accretion rate through the disc, i.e. the main parameter regulating the accretion phase of a YSO, has been measured on samples of CTTSs with a wide range of masses (e.g. Muzerolle et al. 1998a; Natta et al. 2006; Herczeg \& Hillenbrand 2008; Fang et al. 2009). These results were mainly obtained using optical (e.g. Ca II and $\mathrm{H} \alpha$ ) and IR $(\mathrm{Pa} \beta$ and $\mathrm{Br} \gamma)$ emission lines to measure the mass accretion rate, adopting relationships between accretion luminosity and line flux luminosities calibrated on direct tracers of accretion like the excess UV emission over the stellar photosphere (e.g. Gullbring et al. 1998). More limited quantitative studies of this kind have been performed in the IR on embedded Class I sources, for which the stellar parameters are much more difficult to constrain owing to the considerable IR veiling (Nisini et al. 2005b; Antoniucci et al. 2008; Connelley \& Greene 2010).

These previous works were mainly based on observations performed on limited spectral ranges, which sometimes covered only individual diagnostic lines, which in turn made the results dependent on the choice of the tracer. In addition, most studies have been performed on non-homogeneous samples of CTTSs with similar ages, which means that no attempt has been performed to compare samples of YSOs of different ages in starforming regions with different general characteristics.

In this framework, and to derive quantitative indicators of YSO activity, we have undertaken a combined optical/IR unbiased spectroscopic survey on a flux-limited sample of Spitzer selected Class I/II sources hosted in six different nearby clouds (namely Cha I-II, L1641, Ser, Lup, Vel, and CrA), using the EFOSC2/SOFI instruments mounted on the ESO-NTT telescope. A total number of about 150 sources have been observed in a wide wavelength range (from 0.6 to $2.4 \mu \mathrm{m}$ ) at low resolution $(R \sim 700)$ during this project, which has been named POISSON: Protostellar Objects IR-optical Spectral Survey On $N T T$. The present article is the first of a series on the analysis of the POISSON data.

In this paper we report on the observations performed on 47 YSOs in the Chamaeleon I and II molecular clouds $(d=160 \mathrm{pc}$ and 178 pc, Whittet et al. 1997). The Chamaeleon complex consists of three main dark clouds, namely Cha I, Cha II, and Cha III (Schwartz et al. 1991), which present very different starformation efficiencies (SFE). In particular, Cha I is the most active of the three clouds, with an SFE of $\sim 10 \%$ (Luhman 2007; Mizuno et al. 1999), while the SFE estimated for Cha II is only $\sim 1-3 \%$. Observations of the Cha I and II samples will therefore allow us to compare properties of sources located at the same distance, but with a different star-formation activity.

The population of the Cha I/II clouds has been extensively studied in the past (see review by Luhman et al. 2008, and references therein). Recent Spitzer observations have been combined with near-IR photometry to take a complete census of the
YSO population in the two clouds (see e.g. Young et al. 2005; Allers et al. 2006; Porras et al. 2007; Damjanov et al. 2007; Alcalá et al. 2008; Luhman et al. 2005, 2008; Luhman \& Muench 2008). Optical spectroscopic follow-up has then been used to derive the stellar parameters of the identified YSOs (Luhman 2007; Spezzi et al. 2008). The estimated mean age of the stellar population is $2 \mathrm{Myr}$ for Cha I (Luhman et al. 2008) and 3-4 Myr for Cha II (Spezzi et al. 2008). Infrared spectroscopy of a few sources of the Cha I/II clouds have been analysed by Gómez \& Persi (2002) and Natta et al. (2004), while accretion rates for limited sub-samples have been derived using the lumi-

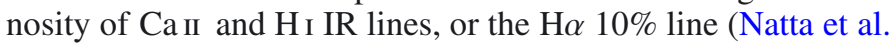
2004; Mohanty et al. 2005; Spezzi et al. 2008).

Taking advantage of this huge and detailed database of information available for the YSO population in Cha I/II, we have investigated here he accretion properties of these sources by using and comparing the existing empirical relationships between line luminosity and accretion luminosity. Thanks to the wide range of wavelengths covered by our observations, we have been able to measure the accretion luminosity $\left(L_{\mathrm{acc}}\right)$ through different tracers in a homogeneous way and, more importantly, to compare the $L_{\text {acc }}$ determinations independently of the source variability. We have therefore focused on the analysis of the results obtained with the different lines, discussing the consistency of the empirical relationships used and the reliability of the considered tracers. Part of the analysis has also focused on the derivation of the excitation conditions pertaining to the IR HI lines, because these lines are the only tracers of accretion that can be used in both CTTSs and embedded sources.

The paper is structured as follows: in Sects. 2 and 3 we introduce the sample and present the main source-properties derived from the literature. In Sect. 4 we describe the observations and data reduction. In Sect. 5 we present the spectra and the detected features. Then, we derive the accretion luminosity of the objects from the different tracers in Sect. 6, comparing and discussing the obtained values. The analysis of the observed $\mathrm{H}_{\text {I }} \mathrm{Pa} \beta / \mathrm{Br} \gamma$ ratio, which provides information on the $\mathrm{H}_{\mathrm{I}}$ emission mechanism, is given in Sect. 8. The main conclusions of our work are finally summarised in Sect. 9.

\section{Sample selection}

The sample has been selected from the young population of ClassI/II objects identified through Spitzer surveys of the Chamaeleon I and II molecular clouds. Reference works were Luhman (2007) and Luhman et al. (2008) for Cha I, and Alcalá et al. (2008) and Spezzi et al. (2008) for Cha II. From these surveys we selected the sources with an SED spectral index between 2 and $24 \mu \mathrm{m} \alpha_{2-24} \gtrsim-1$ and a $K$-band magnitude $<12 \mathrm{mag}$, constrained by instrumental sensitivity. This selection resulted in a total of 29 (15) targets for the Cha I (Cha II) cloud, namely 2 (1) Class I, 4 (0) flat and 23 (14) Class II objects. Three more Class IIs with $\alpha<-1$ ( 1 in Cha I and 2 in Cha II) were found to be very close to some of the selected targets and were accordingly observed with the same slit acquisition. These sources were eventually included in the sample, which therefore amounts to a total of 47 objects.

The very small number of Class I sources is due to the relatively old age of the population of these clouds, with respect to other nearby molecular clouds such as $\rho$ Oph.

The complete list of targets is given in Tables 1 and 2 together with their main parameters, which are presented in the following section. 
Table 1. Main properties of Cha I targets.

\begin{tabular}{|c|c|c|c|c|c|c|c|c|c|c|c|c|}
\hline $\begin{array}{l}\text { Id } \\
\text { Cha I }\end{array}$ & Name & $\alpha^{a}(2000)$ & $\delta^{a}(2000)$ & Other names & $\alpha_{2-24 \mu \mathrm{m}}$ & $\begin{array}{c}L_{\mathrm{IR}}^{b} \\
L_{\odot}\end{array}$ & $\begin{array}{l}L_{*} \\
L_{\odot}\end{array}$ & $\begin{array}{l}R_{*} \\
R_{\odot}\end{array}$ & $\begin{array}{l}M_{*} \\
M_{\odot}\end{array}$ & ST & $\begin{array}{l}A_{V}{ }^{c} \\
\text { mag }\end{array}$ & $\begin{array}{c}m_{K}^{d} \\
\mathrm{mag}\end{array}$ \\
\hline 1 & T11 & $11: 02: 24.91$ & $-77: 33: 35.7$ & Sz9, CS Cha & -0.72 & 0.96 & 1.2 & 2.07 & 0.87 & K6 & 0.21 & 8.20 \\
\hline 2 & CHSM1715 & 11:04:04.25 & $-76: 39: 32.8$ & CHSM1715 & -0.62 & 0.08 & 0.05 & 0.71 & 0.2 & M4.25 & 2.22 & 10.90 \\
\hline 4 & ISO52 & $11: 04: 42.58$ & $-77: 41: 57.7$ & ISO52, B18 & -0.84 & 0.07 & 0.09 & 0.94 & 0.25 & M4 & 1.08 & 10.64 \\
\hline 5 & Hn5 & 11:06:41.80 & $-76: 35: 48.9$ & Hn5 & -0.82 & 0.13 & 0.11 & 1.08 & 0.22 & M4.5 & 0.96 & 10.13 \\
\hline 6 & ISO92 & 11:07:09.19 & $-77: 23: 04.9$ & IRAS11057-7706 & 0.71 & 0.60 & - & - & - & - & $6.0^{f}$ & 10.92 \\
\hline 7 & ISO97 & $11: 07: 16.22$ & $-77: 23: 06.8$ & - & 0.15 & 0.09 & - & - & - & - & $10.0^{f}$ & 11.68 \\
\hline 8 & $\mathrm{~T} 26$ & $11: 07: 20.74$ & $-77: 38: 07.3$ & Sz19, DI Cha & -0.92 & 4.00 & 12 & 3.37 & 2 & $\mathrm{G} 2$ & 2.25 & 6.22 \\
\hline 9 & B35 & $11: 07: 21.42$ & $-77: 22: 11.7$ & ISO101 & -0.05 & 0.18 & - & - & - & - & $20.0^{f}$ & 10.93 \\
\hline 10 & CHXR30B & $11: 07: 57.30$ & $-77: 17: 26.2$ & B38 & -0.79 & 0.24 & 0.22 & 1.16 & 0.58 & M1.25 & 9.47 & 9.95 \\
\hline 11 & T30 & $11: 07: 58.09$ & $-77: 42: 41.3$ & $\mathrm{Sz} 23$ & -0.91 & 0.16 & 0.15 & 1.06 & 0.44 & M2.5 & 3.77 & 9.89 \\
\hline 12 & CHXR30A & 11:08:00.02 & $-77: 17: 30.4$ & CHXR30A & -1.63 & 0.32 & 1.4 & 2.52 & 0.95 & $\mathrm{~K} 8$ & 8.93 & 9.09 \\
\hline 13 & T31 & 11:08:01.48 & $-77: 42: 28.8$ & Sz24, VW Cha & -0.98 & 1.93 & 3 & 3.7 & 0.74 & K8 & 2.16 & 6.96 \\
\hline 14 & Cha IRN & 11:08:38.96 & $-77: 43: 51.3$ & C9-2,ISO150 & $0.84^{e}$ & 2.02 & - & - & - & $<\mathrm{M} 0$ & $11.0^{f}$ & 8.71 \\
\hline 15 & $\mathrm{C} 9-3$ & 11:08:42.96 & $-77: 43: 50.0$ & - & -0.79 & 0.12 & - & - & 0.25 & - & $\sim 0^{f}$ & 11.78 \\
\hline 16 & T38 & 11:08:54.64 & $-77: 02: 12.9$ & Sz29, VY Cha & -0.88 & 0.23 & 0.34 & 1.36 & 0.66 & M0.5 & 2.70 & 9.46 \\
\hline 18 & $\mathrm{C} 1-6$ & $11: 09: 22.66$ & $-76: 34: 32.0$ & CED112-IRS2 & -0.49 & 0.90 & 0.8 & 2.21 & 0.58 & M1.25 & 9.79 & 8.67 \\
\hline 19 & $\mathrm{C} 1-25$ & 11:09:41.92 & $-76: 34: 58.4$ & ISO199 & -0.23 & 0.30 & - & - & - & - & $14.0^{f}$ & 10.00 \\
\hline 20 & Hn10-e & $11: 09: 46.21$ & $-76: 34: 46.3$ & FL2004 55 & -0.46 & 0.15 & 0.15 & 1.13 & 0.34 & M3.25 & 3.02 & 10.05 \\
\hline 21 & B43 & $11: 09: 47.42$ & $-77: 26: 29.0$ & ISO207 & -0.91 & 0.15 & 0.22 & 1.37 & 0.36 & M3.25 & 6.77 & 10.24 \\
\hline 22 & $\mathrm{~T} 42$ & $11: 09: 53.40$ & $-76: 34: 25.5$ & ISO223,Sz32,FM Cha & $-0.55^{e}$ & 3.61 & 3 & 3.05 & 0.75 & K5 & 4.40 & 6.46 \\
\hline 23 & $\mathrm{~T} 43$ & 11:09:54.07 & $-76: 29: 25.3$ & Sz33, CHXX12 & -0.87 & 0.29 & 0.48 & 1.82 & 0.54 & M2 & 4.40 & 9.25 \\
\hline 24 & C1-2 & $11: 09: 55.05$ & $-76: 32: 40.9$ & ISO226, CED112-IRS5 & 0.03 & 0.57 & - & - & - & - & $14.0^{f}$ & 9.67 \\
\hline 25 & Hn11 & 11:10:03.69 & $-76: 34: 58.4$ & ISO232 & -0.64 & 0.36 & 0.66 & 1.73 & 0.76 & K8 & 6.41 & 9.44 \\
\hline 26 & ISO237 & $11: 10: 11.41$ & $-76: 35: 29.2$ & ISO237 & -0.58 & 0.59 & 1.2 & 2 & 0.95 & K5.5 & 5.75 & 8.62 \\
\hline 27 & T47 & $11: 10: 49.59$ & $-77: 17: 51.7$ & Sz37, HBC584 & -0.57 & 0.31 & 0.42 & 1.71 & 0.53 & M2 & 3.50 & 9.18 \\
\hline 28 & ISO256 & $11: 10: 53.59$ & $-77: 25: 00.4$ & - & -0.59 & 0.07 & 0.07 & 0.86 & 0.21 & M4.5 & 7.64 & 11.34 \\
\hline 29 & T49 & $11: 11: 39.65$ & $-76: 20: 15.2$ & Sz39, XX Cha & -0.92 & 0.31 & 0.37 & 1.6 & 0.53 & M2.5 & 1.02 & 8.87 \\
\hline 30 & T53 & $11: 12: 30.92$ & $-76: 44.24 .1$ & Sz43, CW Cha & $-1.05^{e}$ & 0.33 & 0.39 & 1.52 & 0.5 & M1 & 2.37 & 9.13 \\
\hline
\end{tabular}

Notes. Stellar parameters $L_{*}, R_{*}, M_{*}, \mathrm{ST}$ and extinction are taken from Luhman $(2004,2007)$, unless explicitly noted. ${ }^{(a)}$ Coordinates from the 2MASS catalogue. ${ }^{(b)}$ Luminosity computed by integrating the spectral energy distribution between 1 and $70 / 100 \mu \mathrm{m}$, using $2 \mathrm{MASS}$ and Spitzer measurements corrected for extinction. ${ }^{(c)}$ Obtained from $A_{J}$ given by Luhman $(2007) .{ }^{(d)} K$-band magnitude from 2 MASS. ${ }^{(e)}$ Spectral index computed from 2 to $12 \mu \mathrm{m}$ (see Luhman et al. 2008). ${ }^{(f)}$ Extinction estimate from the $(J-H)-(H-K)$ colour-colour diagram.

\section{Source parameters}

\subsection{Spectral type, mass, and luminosity.}

Spectral classification of most of the objects has been performed on the basis of optical spectroscopy by $\operatorname{Luhman}(2004,2007)$ for Cha I and by Spezzi et al. (2008) for Cha II. The spectral types, stellar masses, and luminosities derived by these authors are reported in Tables 1 and 2. Basically, all sources present late spectral types $(\mathrm{K}, \mathrm{M})$ and masses ranging between 0.2 and $1.4 M_{\odot}$, with the exception of the two $2 M_{\odot}$ sources DI Cha (Cha I-8, spectral type G2) and DK Cha (Cha II-1, spectral type F0).

For each source we also computed the total luminosity between 1 and 70 or $100 \mu \mathrm{m}$ (depending on the longest wavelength photometric measurement available), which we indicate as $L_{\mathrm{IR}}$, by integrating the $\mathrm{SED}^{1}$ constructed with photometry from the 2MASS survey (Skrutskie et al. 2006) and Spitzer instruments IRAC and MIPS (see Alcalá et al. 2008; Luhman et al. 2008). We notice that for almost all sources $L_{\mathrm{IR}}$ is very similar or comparable (within a factor of 2) with $L_{*}$. We may interpret this finding as another indication that most of the objects are quite evolved sources characterised by weak infrared excesses and presenting a luminosity that is basically dominated by the stellar photospheric emission. We note that the few sources where $L_{*}$ and $L_{\mathrm{IR}}$ are significantly different display either relatively early spectral types (e.g. Cha I-8) or high extinctions (e.g. Cha II-1, Cha II-3).

\footnotetext{
1 The computation was performed starting from the $J$-band value and considering straight lines (in the $\log (\lambda)-\log \left(\lambda F_{\lambda}\right)$ plan) between available SED points corrected for extinction.
}

The spectral classification, and consequently the relative stellar parameters, were not available for seven objects of the Cha I cloud. Noticeably, these are the reddest sources of our Cha I sample (i.e. displaying the highest values of the spectral index $\alpha_{2-24} \mu \mathrm{m}$ ) and are probably more embedded than the others (see Sect. 3.2). This is consistent with the difficulties Luhman (2004) had to obtain a good quality optical spectrum and derive a spectral type for these objects. In the remainder of the paper we will refer to our estimate of $L_{\mathrm{IR}}$ in place of the unknown $L_{*}$ for these seven sources. However, because these are the objects of the sample characterised by the strongest IR excess, $L_{\mathrm{IR}}$ is likely to overestimate $L_{*}$ in these cases.

\subsection{Extinction}

The knowledge of the amount of extinction toward the sources is fundamental to correctly de-redden line fluxes, which will eventually be used to derive the accretion luminosities (see Sect. 6.2). Extinction estimates for our targets are available in the literature for both Cha I ( $A_{J}$ values from Luhman 2004) and Cha II ( $A_{V}$ values from Spezzi et al. 2008).

Investigations of the interstellar medium in the Chamaeleon regions have shown that the value of the total-to-selective extinction ratio $R_{V}$ is fairly high in both clouds: greater than 5 in Cha I (Luhman 2004) and up to 5-6 in the densest parts of Cha II (e.g. Vrba \& Rydgren 1984; Whittet et al. 1994, 1997). On this basis, we have adopted the extinction law of Cardelli et al. (1989) with $R_{V}=5.5$ to transform the provided $A_{J}$ and $A_{V}$ values to obtain 
Table 2. Main properties of Cha II targets.

\begin{tabular}{|c|c|c|c|c|c|c|c|c|c|c|c|c|}
\hline $\begin{array}{l}\text { Id } \\
\text { Cha II }\end{array}$ & Name & $\alpha^{a}(2000)$ & $\delta^{a}(2000)$ & Other names & $\alpha_{2-24 \mu \mathrm{m}}$ & $\begin{array}{c}L_{\mathrm{IR}}^{b} \\
L_{\odot}\end{array}$ & $\begin{array}{l}L_{*} \\
L_{\odot} \\
\end{array}$ & $\begin{array}{l}R_{*} \\
R_{\odot} \\
\end{array}$ & $\begin{array}{l}M_{*} \\
M_{\odot} \\
\end{array}$ & ST & $\begin{array}{c}A_{V} \\
\text { mag }\end{array}$ & $\begin{array}{c}m_{K}^{c} \\
\mathrm{mag}\end{array}$ \\
\hline 1 & DK Cha & $12: 53: 17.22$ & $-77: 07: 10.6$ & IRAS12496-7650 & -0.72 & 53.7 & 18.62 & 2.77 & $2^{d}$ & F0 & 10.55 & 5.19 \\
\hline 2 & IRAS12535-7623 & $12: 57: 11.72$ & $-76: 40: 11.1$ & CHIIXR2 & -0.98 & 1.16 & 1.38 & 2.71 & 1.05 & M0 & 3.36 & 8.40 \\
\hline 3 & ISO28 & $12: 59: 06.56$ & $-77: 07: 40.1$ & IRAS12553-7651 & 0.56 & 2.29 & 15.85 & 5.58 & $0.7^{d}$ & K4.5 & 38.9 & 10.66 \\
\hline 4 & $\mathrm{Sz} 49$ & $13: 00: 53.23$ & $-76: 54: 15.1$ & ISO55 & -0.61 & 0.19 & 0.2 & 1.03 & 0.75 & M0.5 & 2.28 & 10.63 \\
\hline 5 & Sz48SW & $13: 00: 53.46$ & $-77: 09: 08.6$ & CHIIXR7 & -0.95 & 0.09 & 0.26 & 1.25 & 0.7 & M1 & 3.87 & 9.45 \\
\hline 6 & $\mathrm{Sz} 50$ & 13:00:55.32 & $-77: 10: 22.2$ & ISO52,CHIIXR8 & -0.88 & 0.80 & 1.15 & 3.1 & 0.5 & M3 & 3.78 & 8.85 \\
\hline 7 & CM Cha & $13: 02: 13.51$ & $-76: 37: 57.7$ & CHIIXR13 & -0.95 & 0.83 & 0.72 & 1.78 & 1.15 & K7 & 1.52 & 8.52 \\
\hline 8 & IRAS13005-7633 & $13: 04: 22.84$ & $-76: 50: 05.5$ & $\mathrm{Hn} 22$ & -0.66 & 0.31 & 0.23 & 1.24 & 0.5 & M2 & 0.61 & 9.73 \\
\hline 9 & $\mathrm{Hn} 23$ & $13: 04: 24.10$ & $-76: 50: 01.2$ & - & -1.03 & 0.87 & 0.87 & 1.6 & 1.4 & K5 & 1.24 & 8.77 \\
\hline 10 & $\mathrm{Hn} 24$ & 13:04:55.71 & $-77: 39: 49.5$ & - & -0.97 & 0.76 & 1.05 & 2.37 & 1 & M0 & 2.76 & 8.92 \\
\hline 11 & Sz53 & $13: 05: 12.69$ & $-77: 30: 52.5$ & - & -1.07 & 0.28 & 0.32 & 1.39 & 0.75 & M1 & 3.68 & 9.93 \\
\hline 12 & Sz56 & $13.06: 38.82$ & $-77: 30: 35.2$ & - & -1.08 & 0.20 & 0.34 & 1.78 & 0.3 & M4 & 3.18 & 10.41 \\
\hline 13 & Sz57 & $13: 06: 56.56$ & $-77: 23: 05.4$ & CHIIXR34 & -1.55 & 0.28 & 0.41 & 2.21 & 0.15 & M5 & 3.09 & 9.80 \\
\hline 14 & Sz58 & $13: 06: 57.44$ & $-77: 23: 41.5$ & IRAS13030-7707 & -0.93 & 0.81 & 0.69 & 1.43 & 1.2 & K5 & 3.87 & 8.76 \\
\hline 15 & Sz61 & $13: 08: 06.28$ & $-77: 55: 05.1$ & BM Cha & -0.97 & 1.52 & 1.17 & 1.87 & 1.4 & K5 & 3.13 & 7.95 \\
\hline 16 & IRASF13052-7653NW & 13:09:09.87 & $-77: 09: 43.7$ & - & -1.07 & 0.31 & 0.2 & 1.03 & 0.75 & M0.5 & 2.28 & 9.64 \\
\hline 17 & IRASF13052-7653N & 13:09:10.71 & $-77: 09: 44.3$ & - & -1.17 & 0.20 & 0.34 & 1.49 & 0.62 & M1.5 & 0.41 & 9.07 \\
\hline
\end{tabular}

Notes. Stellar parameters $L_{*}, R_{*}, M_{*}, \mathrm{ST}$ and extinction are taken from Spezzi et al. (2008), unless explicitly noted. ${ }^{(a)}$ Coordinates from the $2 \mathrm{MASS}$ catalogue. ${ }^{(b)}$ Luminosity computed by integrating the Spectral Energy Distribution between 1 and $70-100 \mu$ m, using 2 MASS and Spitzer measurements corrected for extinction. ${ }^{(c)} K$-band magnitude from 2MASS. ${ }^{(d)}$ Mass estimate from D'Antona \& Mazzitelli (1997) evolutionary tracks (see Spezzi et al. 2008).

the extinction at the wavelength of interest. If we had used the standard extinction law of Rieke \& Lebofsky (1985) to derive for example the extinction in the $K$-band from the provided $A_{V}$, we would have obtained a variation of about $20 \%$ for $A_{K}$. On average, the targets display relatively low extinction values $\left(A_{V}<4\right.$ for 14 objects in Cha I and 15 in Cha II, see Tables 1 and 2), which generally agrees with the negative spectral index $\alpha_{2-24} \mu \mathrm{m}$ measured in most of the sources.

No extinction value was given by Luhman for the seven Cha I objects without spectral classification, four of which present $\operatorname{Br} \gamma$ line emission. For these sources we therefore derived a rough estimate of $A_{V}$ from their position on the 2MASS $J-H$ vs. $H-K$ colour-colour diagram. Adopting the extinction law of Cardelli et al. (1989) with $R_{V}=5.5$, we dereddened the objects to bring them back on the CTTS locus (Meyer et al. 1997). We find quite high extinctions $\left(A_{V}>6\right)$ for all sources except for Cha I-15, which conversely presents near-IR colours compatible with an extinction close to zero.

\section{Observations and data reduction}

Our observations were carried out at the ESO New Technology Telescope (Tarenghi \& Wilson 1989) with the EFOSC2 and SofI spectrographs (Morwood 1997; D'Odorico 1988) on 1014 February 2009. We used the EFOSC2 grism \#16 equipped with the 0.7 slit $(R \sim 700)$, and the red and blue grisms (hereafter $\mathrm{RG}$ and $\mathrm{BG}$ ) of SofI in combination with the $0 . ' 6$ slit $(R \sim 900)$. The three low-resolution spectra span the 0.60-1.02, $0.94-1.65$, and $1.50-2.40 \mu \mathrm{m}$ intervals, respectively, and accordingly provide a complete coverage of the $0.6-2.4 \mu \mathrm{m}$ wavelength range.

We acquired the RG spectrum for all sources of the sample, while the BG and EFOSC2 (hereafter "optical") spectra were taken only for the brightest objects with $J<14$ and $I<15$, respectively. Hence, the final dataset consists of $47 \mathrm{RG}, 38 \mathrm{BG}$, and 33 optical spectra (see Tables 3 and 4). All data were reduced using the IRAF ${ }^{2}$ software package.

\footnotetext{
${ }^{2}$ IRAF (Image Reduction and Analysis Facility) is a general-purpose software system for the reduction and analysis of astronomical data.
}

For the infrared spectra we followed the standard procedures for bad pixel removal, flat-fielding, and sky-subtraction. Spectra of telluric standard stars were acquired at air-masses similar to those of the targets and were used after removal of any intrinsic line to correct the scientific spectra for atmospheric absorption. Wavelength calibration for all spectra was obtained using xenonargon arc lamps.

Strong seeing variations and instrumental problems (the source did not remain correctly centred on the slit passing from the A to B positions during the nodding) caused flux losses that prevented us from calibrating the flux scale of the spectra using spectro-photometric standards.

Hence, we adopted the 2MASS $K$-band magnitude for the calibration of the RG spectral segment. Then, we normalised the BG segment to obtain a good match of the two spectra in the spectral region where they overlap. Once this procedure was applied, we checked the consistency of our calibrated spectra with the 2MASS $J$ and $H$ points and found a very good agreement for most of the objects $(\Delta(H-K)$ and $\Delta(J-K) \leq 0.1 \mathrm{mag})$, with maximum colour discrepancies of $\Delta(H-K)=0.3$ and $\Delta(J-K)=0.4 \mathrm{mag}$. This is an indication that there have been no strong variations of the infrared colours of the sources since the 2MASS measurements.

The EFOSC2 spectra were reduced performing the standard procedures for dark current subtraction, bad pixel and fringing removal, and flat-fielding. The wavelength calibration was carried out using helium-argon arc lamps. The spectra acquired with grism \#16 display an evident drop owing to an efficiency loss above about $0.9 \mu \mathrm{m}$. This effect could not be properly calibrated out using spectro-photometric standard star spectra because it appears to be strongly dependent on the colour of the observed source, so that it turns out to be very different for the standards with respect to our scientific targets, which are much redder. We therefore decided to cut the optical spectra at $0.9 \mu \mathrm{m}$. Because of flux losses caused by seeing variation during the acquisitions, we preferred to use the BG spectrum as a reference to flux-calibrate

IRAF is written and supported by the IRAF programming group at the National Optical Astronomy Observatories (NOAO) in Tucson, Arizona. http://iraf. noao.edu 
the optical segment. The agreement we find with available $I$ band photometry is good for the vast majority of the sources and the maximum colour variation we observe is $\Delta(I-K)=0.5 \mathrm{mag}$.

Although the contiguity and partial overlapping of the spectral segments ensures a good inter-calibration of the spectra, the absolute calibration of the flux scale suffers from the uncertainty of the value of the $K$-band magnitude at the moment of observations with respect to the 2 MASS $K$-value, which was eventually used as reference. Observations show that young stellar objects display a $K$-band mean variability that can be estimated to be of the order of $0.5 \mathrm{mag}$ over time scales of years (see e.g. Alves de Oliveira \& Casali 2008). Consequently, we will assume this value, which corresponds to a factor of about 1.6 in flux, as an estimate of the error for the flux calibration of our spectra.

\section{Spectra}

The spectra of the sources of the two samples are displayed in Figs. 1 and 2. They show several emission lines that remain unresolved at the low spectral resolution of the observations.

We detect many permitted emission lines commonly found in young sources: the most numerous features are the $\mathrm{H}$ i recombination lines from the Paschen and Brackett series (in particular $\mathrm{Pa} \beta$ and $\mathrm{Br} \gamma$ ) and $\mathrm{H} \alpha$ at optical wavelengths. $\mathrm{Br} \gamma$ is detected in 39 sources out of the 47 composing the sample. Other permitted lines we observe are the $\mathrm{He}_{\mathrm{I}}$ line at $1.08 \mu \mathrm{m}$, which is commonly associated with emission in expanding stellar winds (e.g. Edwards et al. 2006; Ray et al. 2007), the Ca II triplet (8498, 8542, $8662 \AA$ ), and the $\mathrm{Mg}_{\mathrm{I}}$ at $1.5 \mu \mathrm{m}$.

ChaII-1 (DK Cha) displays the richest emission spectrum in the sample: this is also the only object showing significant emission from $\mathrm{Na}$ I $(2.2 \mu \mathrm{m})$ and ro-vibrational $\mathrm{CO}$ transitions (from $2.3 \mu \mathrm{m}$ on). Both features are usually associated with the presence of active circumstellar discs (e.g. Najita et al. 2003; Antoniucci et al. 2008). This interesting object is analysed in detail in a dedicated paper (García López et al. 2011).

The forbidden emission lines of [O I], [S II], [Fe II] are classical indicators of outflow activity in young stellar objects (e.g. Reipurth \& Bally 2001; Nisini et al. 2005b; Podio et al. 2006). While the [OI] line at $6300 \AA$ is detected in 15 objects, the [S II] emission $(6716+6731 \AA)$ is observed only in three sources and [Fe II] lines (e.g. $1.25 \mu \mathrm{m}, 1.64 \mu \mathrm{m}$ ) are detected only in ChaII-1.

The relative lack of jet-line detections (see Tables 3 and 4) can mainly be ascribed to the low resolution and limited sensitivity of the observations. This is also consistent with the fact that all these lines are indeed observed in the brightest object (Cha II-1). For example, on the basis of the $\left[\mathrm{O}_{\mathrm{I}}\right] /[\mathrm{Fe} \mathrm{II}] \mathrm{ra}-$ tio observed in various jets, which is in the range $0.1-1$ (e.g. Nisini et al. 2005b; Podio et al. 2006), we can expect intrinsic fluxes of the order $10^{-14}-10^{-15} \mathrm{erg} \mathrm{s}^{-1} \mathrm{~cm}^{-2}$ for the [Fe II] $1.25 \mu \mathrm{m}$ line, which will be even lower in the observed spectra owing to the extinction. Now, considering the typical rms we measure in the $J$ band, we find a $(3 \sigma)$ upper limit of about $2 \times 10^{-14} \mathrm{erg} \mathrm{s}^{-1} \mathrm{~cm}^{-2}$, which means that our observations are often not sensitive enough to detect this line.

High spatial resolution observations for $\left[\mathrm{S}_{\mathrm{II}}\right]$ show that the $\left[\mathrm{O}_{\mathrm{I}}\right] /[\mathrm{S}$ II $]$ ratio observed very close to the source in many jetdriving YSOs is usually greater than a factor 3-5 (e.g. Hirth et al. 1997; Melnikov et al. 2009), so that where [O I] is detected at the $10^{-15} \mathrm{erg} \mathrm{s}^{-1} \mathrm{~cm}^{-2}$ level we expect [S $\mathrm{S}_{\mathrm{II}}$ ] fluxes of order $10^{-16} \mathrm{erg} \mathrm{s}^{-1} \mathrm{~cm}^{-2}$, which are again too low for the sensitivity of our survey. In agreement with this picture, we remark that
[S II] emission is observed only in sources displaying an [O I] flux of the order $10^{-14} \mathrm{erg} \mathrm{s}^{-1} \mathrm{~cm}^{-2}$.

The molecular transitions of $\mathrm{H}_{2}$ (in particular the $2.12 \mu \mathrm{m}$ line), which is another typical tracer of shocks from protostellar jets, are detected at a significant level only in four sources. Beck et al. (2008) found extended $\mathrm{H}_{2}$ emission in several bright CTTSs, but the observed lines are characterised by a low contrast against the continuum, even at a spectral resolution of about 5000. Hence, in our sample we are probably not sensitive enough to detect $\mathrm{H}_{2}$ lines, except for the strongest (and/or extended) emission cases. On the other hand, the paucity of $\mathrm{H}_{2}$ features might also suggest a low abundance of molecular gas in the close circumstellar environment of many objects.

In Tables 3 and 4 we report for each source the flux of the five emission lines that will be used in our analysis to derive the accretion luminosity of the objects (namely $\mathrm{H} \alpha, \mathrm{Pa} \beta, \mathrm{Br} \gamma,[\mathrm{O}$ I], and $\mathrm{Ca}$ II, see Sect. 6), providing upper limits for non-detections, and indicating also the presence of other emission features detected at the $3 \sigma$ level.

In general, no evident absorption features are detected in the infrared part of the spectra, as might be expected given both the presence of veiling by excess emission, typically observed in young sources (e.g. Greene \& Lada 1996; Nisini et al. 2005a), and the low resolution of the acquisitions. Conversely, several objects present broad absorption bands ( $\mathrm{TiO})$ at optical wavelengths, which are compatible with late spectral type (late-K and M) photospheres and agree with the classification available in the literature (see Sect. 3).

\section{Determination of accretion properties}

\subsection{Emission lines as accretion tracers}

The wide spectral coverage of our data allows us to observe several emission lines both in the optical and near-IR that can be used to determine the accretion luminosity $\left(L_{\mathrm{acc}}\right)$ of the sources. The use of these lines as accretion tracers relies on the existence of empirical relationships connecting the line luminosity $\left(L_{\text {line }}\right)$ to $L_{\text {acc }}$, which have been established by measuring the accretion luminosity through diagnostics that are independent of the emission lines, such as the UV/blue continuum excess (e.g. Gullbring et al. 1998, 2000; Herczeg \& Hillenbrand 2008). These relationships are typically written in the form

$\log L_{\text {acc }} / L_{\odot}=a \cdot \log L_{\text {line }} / L_{\odot}+b$,

where $a$ and $b$ are parameters derived from fitting the observed luminosities (see e.g. Muzerolle et al. 1998a; Calvet et al. 2000, 2004; Natta et al. 2004; Herczeg \& Hillenbrand 2008; Dahm 2008; Fang et al. 2009). For our analysis we considered in particular five emission lines that are detected in many objects of our sample: [O I] $\lambda 6300, \mathrm{Ca}$ II $\lambda 8542$, and the $\mathrm{H}_{\mathrm{I}}$ transitions $\mathrm{H} \alpha, \mathrm{Pa} \beta$, and $\mathrm{Br} \gamma$. The line luminosity- $L_{\text {acc }}$ relationships we employed are presented and briefly discussed in the Appendix. We remark here that these relations are all substantially based on observations of young sources of the Taurus-Auriga complex.

The simultaneous detection of all or some of these five emission features allows us to derive accretion luminosities that are not biased by intrinsic line flux variations that may occur over long periods of time, as is the case when dealing with data acquired in different observing runs. In our survey the time span between observations of the same source with EFOSC2 and SofI has only been $24-48 \mathrm{~h}$, hence our results are not biased by possible variations of the line flux on timescales longer than this interval. Emission line variability on shorter timescales (hours) 
A\&A 534, A32 (2011)

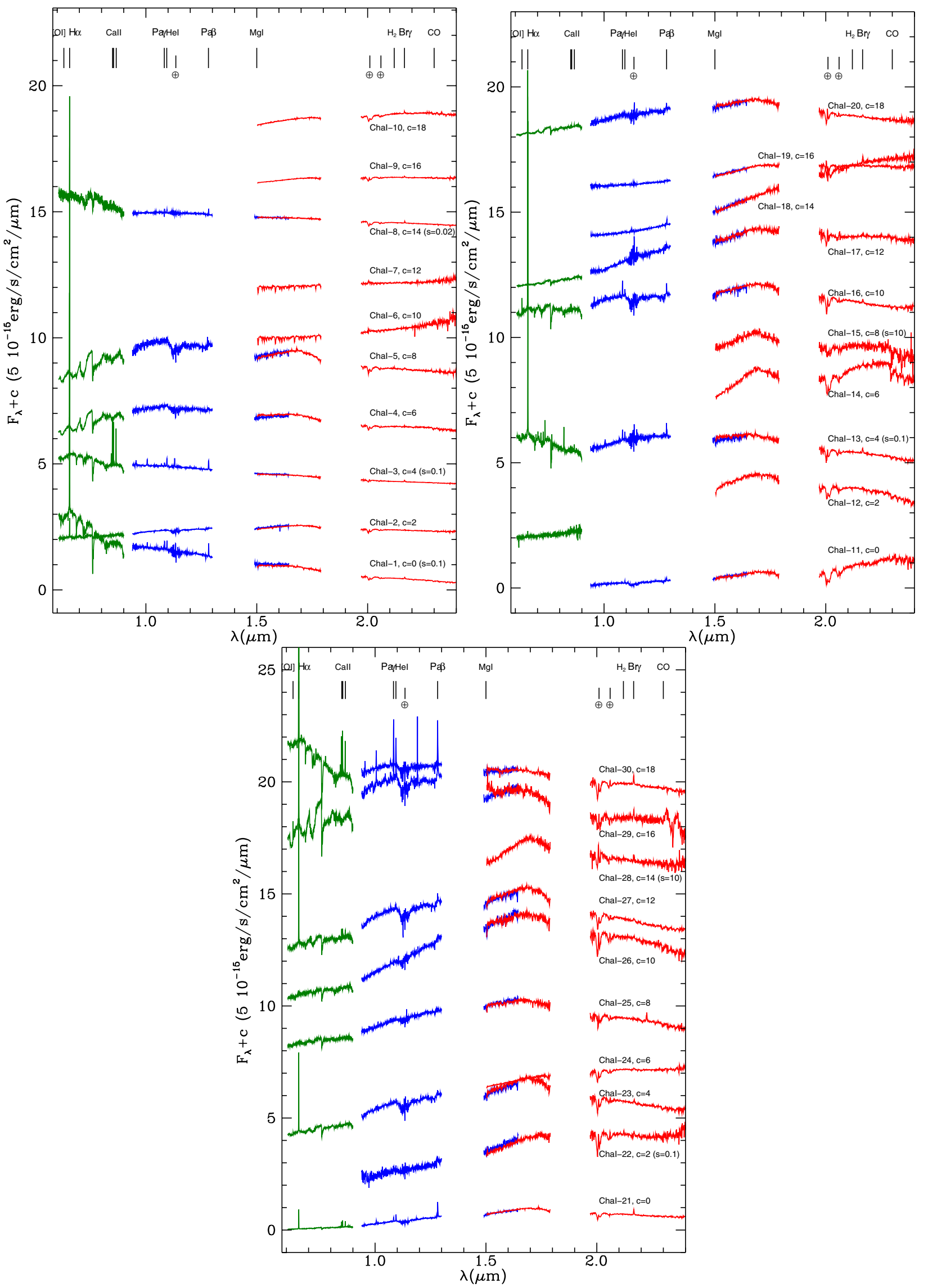

Fig. 1. Spectra of the Cha I sources. The three parts of the spectrum are displayed in different colours (EFOSC2 grism \#16 in green, SofI blue grism in blue, and SofI red grism in red). The spectra were offset and some of them were multiplied by a scale factor $s$ (indicated in parentheses) for better visualisation. Wavelength intervals heavily corrupted by atmospheric absorption were removed. The position of the main emission lines present in the covered spectral range is indicated. 
S. Antoniucci et al.: POISSON observations: emission lines as accretion tracers in YSOs

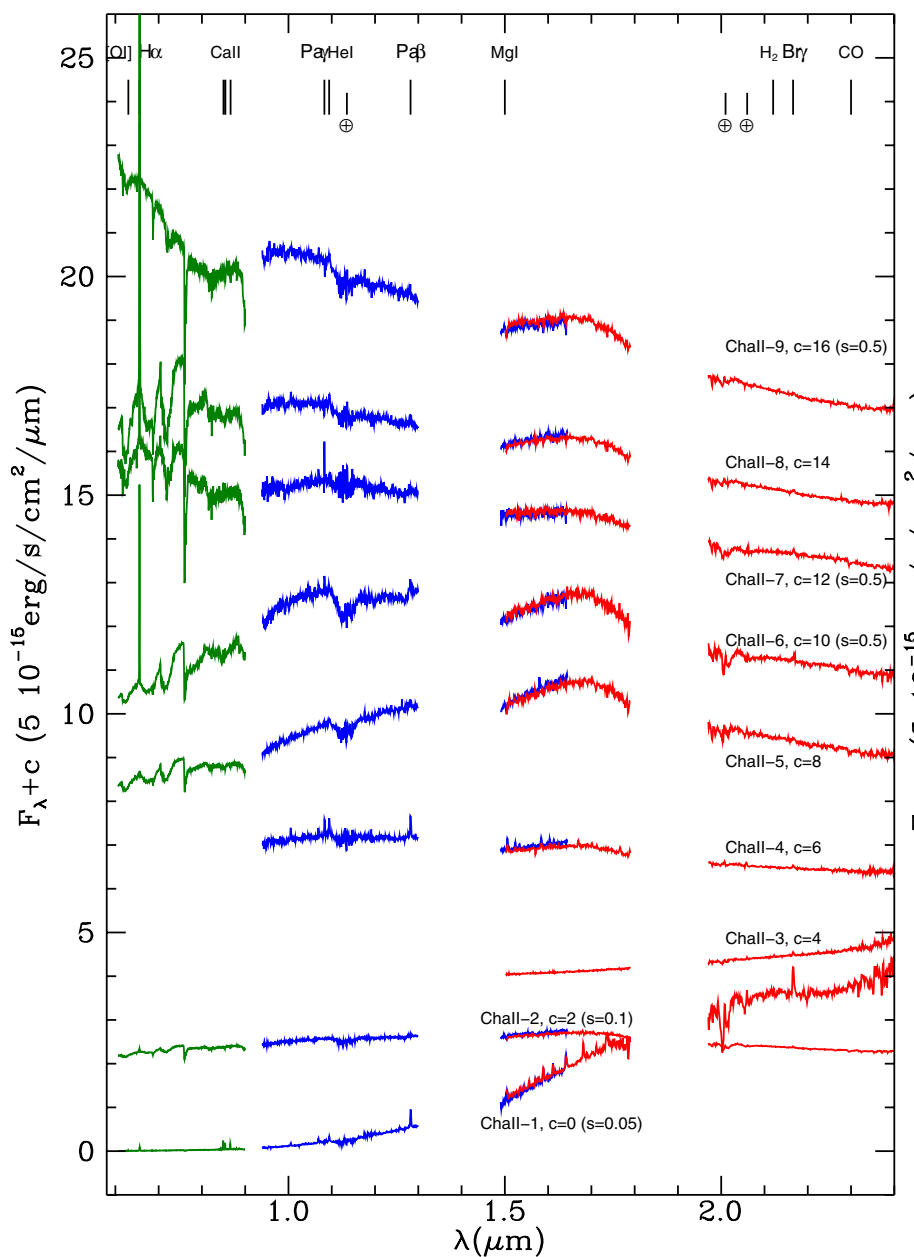

Fig. 2. Spectra of the Cha II sources (see caption of Fig. 1).

has been observed in several young objects: variations affect in particular the line profiles, but the line flux changes at maximum by a factor of a few, which is much less than variations observed on longer timescales (e.g. Scholz et al. 2005; Stelzer et al. 2007; Mendigutía et al. 2011).

Apart from this limitation, the accretion luminosities derived from the five tracers should therefore be consistent with each other. This offers us the opportunity to compare the $L_{\mathrm{acc}}$ determinations and discuss the consistency and reliability of the different relationships employed.

In general, there will be a mix of mechanisms at work in different zones of the circumstellar region that contribute to the line formation, which are more or less directly related to the ongoing accretion process. Indeed, there is evidence that some emission lines do not originate in accretion funnels (at least not only), but rather in the disc, winds, and jets, i.e. they are indirect tracers of the accretion process. For example, $\left[\mathrm{O}_{\mathrm{I}}\right]$ is believed to form in winds/jets driven by accreting sources (e.g. Cabrit et al. 1990; Hartigan et al. 1995). Ca II emission has also been detected in jets (e.g. Nisini et al. 2005b; Podio et al. 2006), indicating that part of the line might originate outside the magnetospheric accretion region. Winds appear to strongly contribute also to $\mathrm{H} \alpha$ (e.g. Reipurth et al. 1996; Calvet et al. 1992). Spatially extended emission suggesting a contribution from a wind is observed also for $\mathrm{H}_{\mathrm{I}}$ infrared lines (e.g. Beck et al. 2010), although in this case the emitting region is expected to be more compact with respect to the other tracers, because of the higher excitation conditions of these lines, especially in the case of $\operatorname{Br} \gamma$.

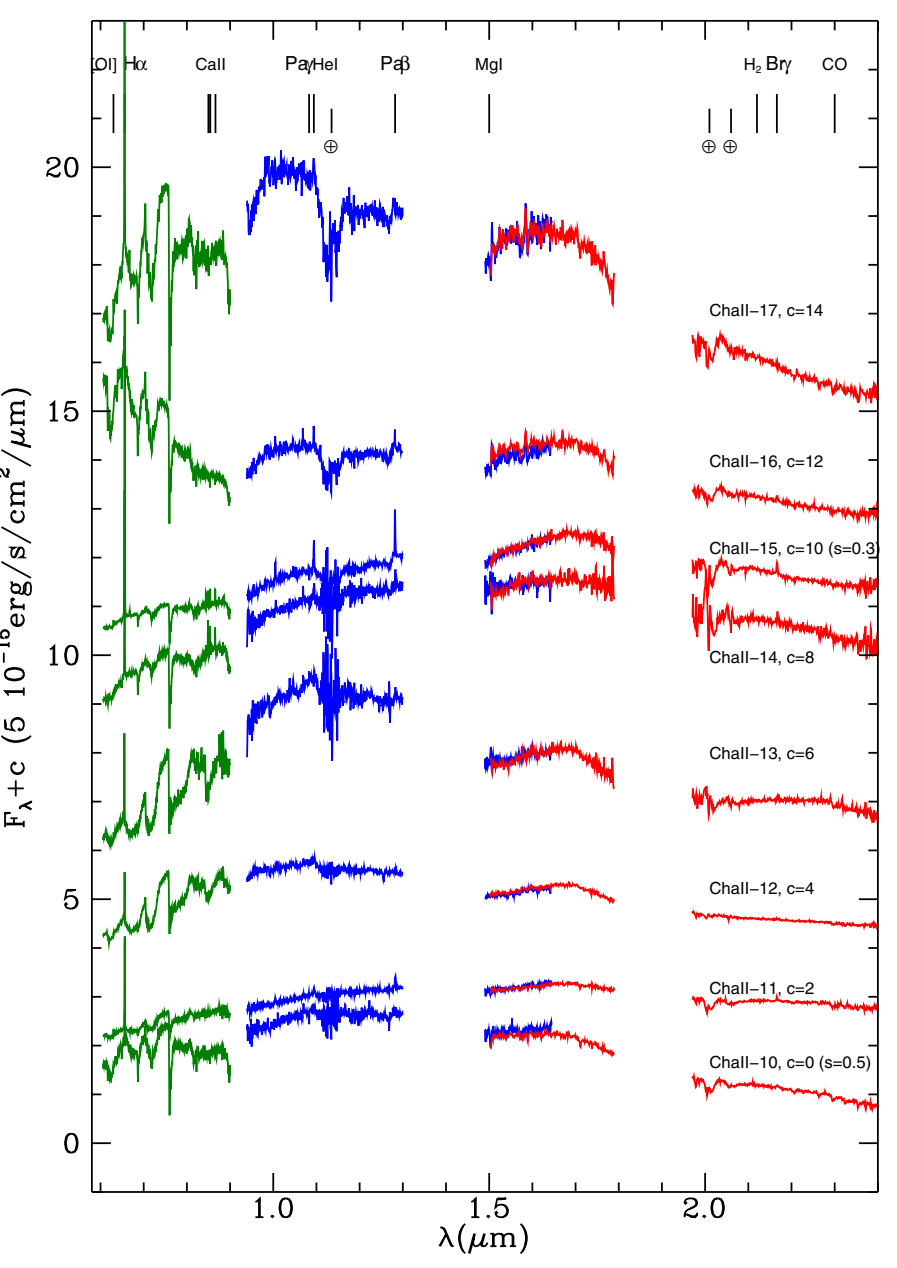

Nevertheless, we point out that the line- $L_{\text {acc }}$ relationships represent an empirical result, so that they do not strictly depend on the actual physical mechanism regulating the accretion. The comparison between the various $L_{\text {acc }}$ determinations obtained in other samples than those over which the relations have been calibrated can therefore reveal particular trends and/or systematic biases of these empirical formulas, thus indicating the tracers that are less affected by these problems.

\subsection{Accretion luminosities from tracers: comparison and discussion}

The line luminosities derived from the measured fluxes (correcting for extinction and distance) and the accretion luminosities obtained through the formulas given in the appendix are listed in Tables 5 and 6. Upper limits are provided for non-detections.

No flux correction was applied to take into account the stellar photospheric absorption for the lines considered, because this contribution is usually negligible for late spectral types. The effect of this correction on $\mathrm{Pa} \beta$ and $\mathrm{Br} \gamma$ is examined in the case of Cha II-1 (spectral type F0) by García López et al. (2011), who accordingly obtain slightly higher accretion luminosities (9.5 and $8.1 L_{\odot}$, respectively).

First of all, we note that in many sources the tracers provide very different $L_{\text {acc }}$ values. Indeed, we obtain accretion luminosities spanning up to two orders of magnitude in ChaI-12 and ChaII-1 for example. Apart from these considerable fluctuations regarding single sources, it is interesting to analyse the 
Table 3. Cha I sample: measured flux (not corrected for extinction) of the five emission lines used as accretion tracers.

\begin{tabular}{|c|c|c|c|c|c|c|c|c|c|c|c|c|c|}
\hline $\begin{array}{l}\text { Id } \\
\text { Cha I }\end{array}$ & $\begin{array}{c}\text { Spectral } \\
\text { segment }^{a}\end{array}$ & $\begin{array}{r}{\left[\mathrm{O}_{\mathrm{I}}\right] 0.630} \\
(F \pm \Delta F) 10^{-14} \\
\mathrm{erg} \mathrm{s}^{-1} \mathrm{~cm}^{-2}\end{array}$ & $\begin{array}{c}\mathrm{m} \\
E W \\
\AA\end{array}$ & $\begin{array}{r}\mathrm{H} \alpha \\
(F \pm \Delta F) 10^{-14} \\
\mathrm{erg} \mathrm{s}^{-1} \mathrm{~cm}^{-2}\end{array}$ & $\begin{array}{c}E W \\
\AA\end{array}$ & $\begin{array}{c}\text { Ca II } 0.854 \\
(F \pm \Delta F) 10^{-14} \\
\mathrm{erg} \mathrm{s}^{-1} \mathrm{~cm}^{-2}\end{array}$ & $\begin{array}{c}\mathrm{m} \\
E W \\
\AA\end{array}$ & $\begin{array}{c}\mathrm{Pa} \beta \\
(F \pm \Delta F) 10^{-14} \\
\mathrm{erg} \mathrm{s}^{-1} \mathrm{~cm}^{-2}\end{array}$ & $\begin{array}{c}E W \\
\AA\end{array}$ & $\begin{array}{c}\mathrm{Br} \gamma \\
(F \pm \Delta F) 10^{-14} \\
\mathrm{erg} \mathrm{s}^{-1} \mathrm{~cm}^{-2}\end{array}$ & $\begin{array}{c}E W \\
\AA\end{array}$ & other lines ${ }^{b}$ & $R$ \\
\hline 1 & OBR & $<5.7$ & & $903.0 \pm 4.8$ & -57.9 & $<12.4$ & & $44.3 \pm 1.7$ & -6.6 & $12.7 \pm 0.9$ & -5.8 & $\mathrm{He}_{\mathrm{I}}$ & $3.6 \pm 0.3$ \\
\hline 2 & OBR & $0.25 \pm 0.07$ & -6.4 & $5.8 \pm 0.1$ & -81.9 & $0.5 \pm 0.1$ & -4.2 & $<0.38$ & & $0.4 \pm 0.1$ & -2.1 & $\mathrm{He}_{\mathrm{I}}$ & $<1.4$ \\
\hline 3 & OBR & $<3.0$ & & $393.0 \pm 1.4$ & -56.4 & $2.0 \pm 0.7$ & -23.4 & $42.9 \pm 1.2$ & -11.1 & $11.8 \pm 0.6$ & -8.2 & $\mathrm{He}_{\mathrm{I}}$ & $4.6 \pm 0.3$ \\
\hline 4 & OBR & $<0.20$ & & $1.8 \pm 0.2$ & -7.7 & $<0.79$ & & $2.2 \pm 0.8$ & -3.9 & $0.3 \pm 0.1$ & & & $8.8 \pm 4.2$ \\
\hline 5 & OBR & $<0.24$ & & $14.7 \pm 0.1$ & -50.2 & $<0.71$ & & $7.2 \pm 0.1$ & -1.0 & $1.8 \pm 0.5$ & -4.8 & $\mathrm{H}_{2}$ & $4.8 \pm 1.2$ \\
\hline 6 & $--\mathrm{R}$ & $\ldots$ & $\ldots$ & $\ldots$ & $\ldots$ & $\ldots$ & $\ldots$ & $\ldots$ & $\ldots$ & $<1.6$ & & & $\ldots$ \\
\hline 7 & $--\mathrm{R}$ & $\ldots$ & $\ldots$ & $\ldots$ & $\ldots$ & $\ldots$ & $\ldots$ & $\ldots$ & $\ldots$ & $<1.1$ & & & $\ldots$ \\
\hline 8 & OBR & $<47.7$ & & $361.0 \pm 15.7$ & -14.3 & $<56.2$ & & $101.0 \pm 5.6$ & -4.6 & $49.6 \pm 3.7$ & -3.6 & $\mathrm{He}_{\mathrm{I}}$ & $3.0 \pm 0.3$ \\
\hline 9 & $--\mathrm{R}$ & $\ldots$ & $\ldots$ & $\ldots$ & $\ldots$ & $\ldots$ & $\ldots$ & $\ldots$ & $\ldots$ & $1.1 \pm 0.2$ & -6.0 & & $\ldots$ \\
\hline 10 & $--\mathrm{R}$ & $\ldots$ & $\ldots$ & $\ldots$ & $\ldots$ & $\ldots$ & $\ldots$ & $\ldots$ & $\ldots$ & $1.7 \pm 0.2$ & -3.8 & & $\ldots$ \\
\hline 11 & $-B R$ & $\ldots$ & $\ldots$ & $\ldots$ & $\ldots$ & $\ldots$ & $\ldots$ & $3.5 \pm 0.4$ & -8.4 & $2.6 \pm 0.5$ & -5.4 & & $2.6 \pm 0.6$ \\
\hline 12 & $\mathrm{O}-\mathrm{R}$ & $0.4 \pm 0.1$ & -46.0 & $1.2 \pm 0.1$ & -47.7 & $1.2 \pm 0.2$ & -10.9 & ... & $\ldots$ & $2.6 \pm 1.1$ & -2.6 & & $\ldots$ \\
\hline 13 & OBR & $7.6 \pm 1.4$ & -1.0 & $557.0 \pm 3.9$ & -67.2 & $1.3 \pm 3.2$ & -2.0 & $48.2 \pm 3.3$ & -4.7 & $46.9 \pm 4.7$ & -6.8 & $\mathrm{He}$ I, [S II] & $1.5 \pm 0.2$ \\
\hline 14 & $--\mathrm{R}$ & $\ldots$ & $\ldots$ & $\ldots$ & $\ldots$ & $\ldots$ & $\ldots$ & $\ldots$ & $\ldots$ & $3.8 \pm 1.6$ & -2.7 & & $\ldots$ \\
\hline 15 & $--\mathrm{R}$ & $\ldots$ & $\ldots$ & $\ldots$ & $\ldots$ & $\ldots$ & $\ldots$ & $\ldots$ & $\ldots$ & $<0.57$ & & & $\ldots$ \\
\hline 16 & OBR & $2.3 \pm 0.1$ & -6.3 & $41.3 \pm 0.2$ & -89.8 & $1.1 \pm 0.1$ & -2.7 & $4.1 \pm 0.3$ & -3.8 & $3.5 \pm 0.4$ & -5.1 & $\mathrm{He}_{\mathrm{I}},\left[\mathrm{S}_{\mathrm{II}}\right]$ & $1.9 \pm 0.2$ \\
\hline 17 & OBR & $0.08 \pm 0.02$ & -2.2 & $0.9 \pm 0.1$ & -15.5 & $<0.27$ & & $1.2 \pm 0.2$ & -1.6 & $3.6 \pm 1.2$ & -3.5 & & $0.9 \pm 0.3$ \\
\hline 18 & $-\mathrm{BR}$ & $\ldots$ & $\ldots$ & $\ldots$ & $\ldots$ & $\ldots$ & $\ldots$ & $3.8 \pm 0.3$ & -10.7 & $5.9 \pm 1.0$ & -4.0 & $\mathrm{He}_{\mathrm{I}}, \mathrm{Mg}_{\mathrm{I}}$ & $3.4 \pm 0.6$ \\
\hline 19 & $-B R$ & $\ldots$ & $\ldots$ & $\ldots$ & $\ldots$ & $\ldots$ & $\ldots$ & $<0.55$ & & $1.6 \pm 0.4$ & -3.8 & & $<3.6$ \\
\hline 20 & OBR & $<0.10$ & & $9.4 \pm 0.1$ & -107.2 & $0.9 \pm 0.1$ & -5.2 & $1.4 \pm 0.2$ & -2.5 & $2.2 \pm 0.4$ & -5.4 & & $1.1 \pm 0.3$ \\
\hline 21 & OBR & $0.07 \pm 0.02$ & -2.1 & $8.5 \pm 0.1$ & -181.5 & $2.4 \pm 0.1$ & -23.3 & $5.8 \pm 0.1$ & -20.7 & $4.0 \pm 0.2$ & -11.8 & $\mathrm{He}_{\mathrm{I}}, \mathrm{Mg}_{\mathrm{I}}$ & $4.5 \pm 0.2$ \\
\hline 22 & $-B R$ & $\ldots$ & $\ldots$ & $\ldots$ & $\ldots$ & $\ldots$ & $\ldots$ & $50.7 \pm 22.0$ & -8.5 & $37.0 \pm 8.1$ & -3.4 & $\mathrm{He} \mathrm{I}_{1} \mathrm{H}_{2}$ & $2.9 \pm 1.4$ \\
\hline 23 & OBR & $0.3 \pm 0.1$ & -2.0 & $19.0 \pm 0.1$ & -90.4 & $0.5 \pm 0.1$ & -1.7 & $1.1 \pm 0.2$ & -1.1 & $4.1 \pm 0.6$ & -4.9 & $\mathrm{He}_{\mathrm{I}}$ & $0.6 \pm 0.1$ \\
\hline 24 & $--\mathrm{R}$ & $\ldots$ & $\ldots$ & $\ldots$ & $\ldots$ & $\ldots$ & $\ldots$ & $\ldots$ & $\ldots$ & $2.1 \pm 0.5$ & -3.7 & & $\ldots$ \\
\hline 25 & OBR & $<0.16$ & & $0.3 \pm 0.1$ & -2.9 & $<0.37$ & & $0.9 \pm 0.3$ & -1.4 & $<2.2$ & & & $>1.2$ \\
\hline 26 & OBR & $<0.32$ & & $0.8 \pm 0.1$ & -3.6 & $<0.61$ & & $<3.3$ & & $3.6 \pm 1.6$ & -2.4 & $\mathrm{He}_{\mathrm{I}}$ & $<2.5$ \\
\hline 27 & OBR & $0.24 \pm 0.07$ & -1.0 & $22.4 \pm 0.2$ & -75.7 & $2.2 \pm 0.2$ & -6.2 & $2.2 \pm 0.4$ & -1.8 & $4.1 \pm 0.9$ & -4.6 & & $0.9 \pm 0.3$ \\
\hline 28 & $--\mathrm{R}$ & $\ldots$ & $\ldots$ & $\ldots$ & $\ldots$ & $\ldots$ & $\ldots$ & $\ldots$ & $\ldots$ & $0.5 \pm 0.1$ & -3.8 & & $\ldots$ \\
\hline 29 & OBR & $3.9 \pm 0.2$ & -5.6 & $86.7 \pm 0.9$ & -86.3 & $<1.2$ & & $13.2 \pm 0.6$ & -6.1 & $6.7 \pm 1.2$ & -5.6 & $\mathrm{He}_{\mathrm{I}}$ & $2.3 \pm 0.4$ \\
\hline 30 & OBR & $<0.28$ & & $48.0 \pm 0.2$ & -69.8 & $3.9 \pm 0.1$ & -9.5 & $19.5 \pm 0.5$ & -14.1 & $7.1 \pm 0.4$ & -7.5 & $\mathrm{He}_{\mathrm{I}}$ & $4.1 \pm 0.2$ \\
\hline
\end{tabular}

Notes. Equivalent widths are given as useful reference. The observed spectral segments are indicated in the second column. Upper limits are provided for non-detections ( $3 \sigma$ level), whereas the "..." indicates that the relative spectral segment is not available. Other emission features detected are also indicated. The last column shows the value of the extinction-corrected $\mathrm{Pa} \beta / \mathrm{Br} \gamma$ ratio $R .{ }^{(a)} \mathrm{O}$ : EFOSC2 grism\#16 optical spectrum; B: SofI blue grism spectrum; R: SofI red grism spectrum. ${ }^{(b)} \mathrm{He}_{\mathrm{I}}$ at $1.08 \mu \mathrm{m}, \mathrm{Mg}_{\mathrm{I}}$ at $1.5 \mu \mathrm{m}, \mathrm{Na}$ at $2.2 \mu \mathrm{m},\left[\mathrm{S}\right.$ II] at $0.67 \mu \mathrm{m}, \mathrm{H}_{2}$ at $2.12 \mu \mathrm{m}$, $\mathrm{CO}(\Delta v=2)$ bandheads longward of $2.23 \mu \mathrm{m}$.

Table 4. Cha II sample: measured flux (not corrected for extinction) of the five emission lines used as accretion tracers (see caption of Table 3).

\begin{tabular}{|c|c|c|c|c|c|c|c|c|c|c|c|c|c|}
\hline \multirow{2}{*}{$\begin{array}{l}\text { Id } \\
\text { Cha II }\end{array}$} & \multirow{2}{*}{$\begin{array}{r}\text { Spectral } \\
\text { segment }^{a}\end{array}$} & \multicolumn{2}{|c|}{ [O I] $0.630 \mu \mathrm{m}$} & \multicolumn{2}{|l|}{$\mathrm{H} \alpha$} & \multicolumn{2}{|c|}{ Са II $0.854 \mu \mathrm{m}$} & \multicolumn{2}{|l|}{$\mathrm{Pa} \beta$} & \multicolumn{2}{|l|}{$\mathrm{Br} \gamma$} & \multirow[t]{2}{*}{ Other lines ${ }^{b}$} & \multirow[t]{2}{*}{$R$} \\
\hline & & $\begin{array}{c}(F \pm \Delta F) 10^{-14} \\
\mathrm{erg} \mathrm{s}^{-1} \mathrm{~cm}^{-2}\end{array}$ & $\begin{array}{c}E W \\
\AA\end{array}$ & $\begin{array}{l}(F \pm \Delta F) 10^{-14} \\
\mathrm{erg} \mathrm{s}^{-1} \mathrm{~cm}^{-2}\end{array}$ & $\begin{array}{c}E W \\
\AA\end{array}$ & $\begin{array}{c}(F \pm \Delta F) 10^{-14} \\
\operatorname{erg~s}^{-1} \mathrm{~cm}^{-2}\end{array}$ & $\begin{array}{c}E W \\
\AA\end{array}$ & $\begin{array}{c}(F \pm \Delta F) 10^{-14} \\
\operatorname{erg~s}^{-1} \mathrm{~cm}^{-2}\end{array}$ & $\begin{array}{c}E W \\
\AA\end{array}$ & $\begin{array}{c}(F \pm \Delta F) 10^{-14} \\
\mathrm{erg} \mathrm{s}^{-1} \mathrm{~cm}^{-2}\end{array}$ & $\begin{array}{c}E W \\
\AA\end{array}$ & & \\
\hline 1 & OBR & $1.6 \pm 0.1$ & -30.3 & $9.3 \pm 0.2$ & -135.8 & $16.9 \pm 0.1$ & -53.9 & $44.3 \pm 0.9$ & -16.9 & $150.0 \pm 5.8$ & -8.8 & $\mathrm{He}_{\mathrm{I}}, \mathrm{H}_{2}, \mathrm{CO}, \mathrm{Na}_{\mathrm{I}}, \mathrm{Mg}_{\mathrm{I}}$ [ $\left.\mathrm{S}_{\mathrm{II}}\right],[\mathrm{Fe}$ II] & $1.9 \pm 0.1$ \\
\hline 2 & OBR & $<1.21$ & & $3.4 \pm 0.2$ & -2.2 & $<3.6$ & & $<6.1$ & & $5.0 \pm 1.0$ & -2.7 & & $<2.1$ \\
\hline 3 & $--\mathrm{R}$ & $\ldots$ & $\ldots$ & $\ldots$ & $\ldots$ & $\ldots$ & $\ldots$ & $\ldots$ & $\ldots$ & $1.4 \pm 0.2$ & -5.9 & $\mathrm{H}_{2}$ & $\ldots$ \\
\hline 4 & $-B R$ & $\ldots$ & $\ldots$ & $\ldots$ & $\ldots$ & $\ldots$ & $\ldots$ & $6.3 \pm 0.3$ & -10.7 & $1.6 \pm 0.3$ & -6.8 & $\mathrm{Mg}_{\mathrm{I}}$ & $5.8 \pm 1.0$ \\
\hline 5 & OBR & $<0.18$ & & $0.4 \pm 0.1$ & -1.8 & $<0.50$ & & $0.9 \pm 0.2$ & -1.1 & $2.6 \pm 1$ & -3.7 & $\mathrm{He}_{\mathrm{I}}$ & $0.7 \pm 0.3$ \\
\hline 6 & OBR & $<0.58$ & & $49.5 \pm 0.2$ & -66.0 & $3.5 \pm 0.5$ & -2.7 & $1.8 \pm 0.3$ & -0.8 & $7.0 \pm 1.1$ & -5.8 & & $0.5 \pm 0.1$ \\
\hline 7 & OBR & $<1.6$ & & $81.6 \pm 0.4$ & -28.8 & $3.2 \pm 0.4$ & -1.6 & $<5.0$ & & $4.0 \pm 0.7$ & -2.4 & & $<1.6$ \\
\hline 8 & OBR & $<1.1$ & & $39.1 \pm 0.2$ & -33.9 & $<1.2$ & & $3.0 \pm 0.7$ & -2.5 & $1.8 \pm 0.4$ & -3.4 & & $1.8 \pm 0.6$ \\
\hline 9 & OBR & $<1.4$ & & $12.9 \pm 0.3$ & -2.5 & $<4.1$ & & $2.8 \pm 0.5$ & -0.9 & $4.2 \pm 1.3$ & -3.3 & & $0.8 \pm 0.3$ \\
\hline 10 & OBR & $<0.89$ & & $6.4 \pm 0.5$ & -3.1 & $<2.8$ & & $<4.0$ & & $3.9 \pm 1.1$ & -3.5 & & $<1.6$ \\
\hline 11 & OBR & $0.7 \pm 0.1$ & -5.0 & $13.9 \pm 0.2$ & -63.8 & $1.3 \pm 0.2$ & -3.4 & $3.8 \pm 0.5$ & -5.9 & $1.8 \pm 0.3$ & -3.9 & & $4.0 \pm 0.9$ \\
\hline 12 & OBR & $0.21 \pm 0.07$ & -1.6 & $4.9 \pm 0.1$ & -16.4 & $<0.92$ & & $1.9 \pm 1.0$ & -2.4 & $0.8 \pm 0.3$ & -2.9 & & $3.8 \pm 2.4$ \\
\hline 13 & OBR & $0.4 \pm 0.1$ & -3.7 & $7.6 \pm 0.2$ & -29.4 & $<2.0$ & & $<3.6$ & & $1.8 \pm 0.4$ & -3.6 & $\mathrm{He}_{\mathrm{I}}$ & $<3.3$ \\
\hline 14 & OBR & $<0.26$ & & $15.9 \pm 0.1$ & -30.4 & $2.8 \pm 0.2$ & -4.1 & $2.5 \pm 0.1$ & -1.7 & $2.7 \pm 0.4$ & -2.1 & & $1.8 \pm 0.3$ \\
\hline 15 & OBR & $1.3 \pm 0.2$ & -1.5 & $127.0 \pm 1.0$ & -114.7 & $5.1 \pm 0.3$ & -3.7 & $31.0 \pm 0.6$ & -8.1 & $13.8 \pm 1.2$ & -5.0 & $\mathrm{He}_{\mathrm{I}}$ & $3.8 \pm 0.3$ \\
\hline 16 & OBR & $<0.50$ & & $1.5 \pm-0.2$ & -1.1 & $<1.0$ & & $2.8 \pm 0.4$ & -2.7 & $1.8 \pm 0.4$ & -3.1 & & $2.3 \pm 0.6$ \\
\hline 17 & OBR & $0.8 \pm 0.1$ & -1.0 & $34.0 \pm 0.2$ & -26.4 & $1.8 \pm-0.3$ & -1.5 & $<7.2$ & & $<2.4$ & & & $\ldots$ \\
\hline
\end{tabular}

Notes. ${ }^{(a)}$ O: EFOSC2 grism\#16 optical spectrum; B: SofI Blue Grism spectrum; R: SofI Red Grism spectrum. ${ }^{(b)} \mathrm{He}$ I at $1.08 \mu \mathrm{m}, \mathrm{Mg}$ I at $1.5 \mu \mathrm{m}$, $\mathrm{Na}$ I at $2.2 \mu \mathrm{m}$, [S II $]$ at $0.67 \mu \mathrm{m},\left[\mathrm{Fe}\right.$ II] at $1.25 \mu \mathrm{m}$ or $1.64 \mu \mathrm{m}, \mathrm{H}_{2}$ at $2.12 \mu \mathrm{m}, \mathrm{CO}(\Delta v=2)$ ro-vibrational bands longward of $2.23 \mu \mathrm{m}$.

general differences of the results derived from the various tracers. To this aim, we plotted in Fig. 3 the $L_{\text {acc }}$ obtained from the five lines as a function of $L_{*}$.

All tracers substantially indicate that $L_{\text {acc }}$ increases with $L_{*}$. However, the plots clearly show that the accretion luminosities derived from the five relationships are actually characterised by very different scatters for given values of $L_{*}$.
In particular, the $\operatorname{Br} \gamma$ line provides the least scattered $L_{\text {acc }}$ values, which basically fall in the range $0.1 L_{*}<L_{\text {acc }}<L_{*}$ for any $L_{*}$, and accordingly result in a quite tight correlation between $L_{\text {acc }}$ and $L_{*} . \mathrm{Pa} \beta, \mathrm{Ca}$ II, and [O I] accretion luminosities display a larger dispersion of about two orders of magnitude, which in the case of $\mathrm{H} \alpha$ goes up to more than three orders of magnitude. Previous works using infrared $\mathrm{H}_{\mathrm{I}}$ emission lines as tracers have 
Table 5. Line luminosities of the five emission tracers considered and accretion luminosities derived using the relevant empirical relationships (see appendix) for Cha I objects.

\begin{tabular}{|c|c|c|c|c|c|c|c|c|c|c|c|}
\hline $\begin{array}{l}\text { Id } \\
\text { Cha I }\end{array}$ & $\begin{array}{c}L_{[\mathrm{O}] \mathrm{I}} \\
L_{\odot} \\
\end{array}$ & $\begin{array}{c}L_{\text {Ca II }} \\
L_{\odot} \\
\end{array}$ & $\begin{array}{c}L_{\mathrm{H} \alpha} \\
L_{\odot} \\
\end{array}$ & $\begin{array}{c}L_{\mathrm{Pa} \beta} \\
L_{\odot} \\
\end{array}$ & $\begin{array}{c}L_{\mathrm{Br} \gamma} \\
L_{\odot} \\
\end{array}$ & $\begin{array}{c}L_{\mathrm{acc}[\mathrm{O}] \mathrm{I}} \\
L_{\odot} \\
\end{array}$ & $\begin{array}{c}L_{\text {accCa II }} \\
L_{\odot} \\
\end{array}$ & $\begin{array}{c}L_{\mathrm{accH} \alpha} \\
L_{\odot} \\
\end{array}$ & $\begin{array}{c}L_{\mathrm{accPa} \beta} \\
L_{\odot} \\
\end{array}$ & $\begin{array}{c}L_{\mathrm{accBr} \gamma} \\
L_{\odot} \\
\end{array}$ & $\begin{array}{l}\dot{M}_{\mathrm{accBr} \gamma} \\
M_{\odot} \mathrm{yr}^{-1} \\
\end{array}$ \\
\hline 1 & $<5.39 \times 10^{-5}$ & $<1.11 \times 10^{-4}$ & $8.49 \times 10^{-3}$ & $3.62 \times 10^{-4}$ & $1.04 \times 10^{-4}$ & $<5.04 \times 10^{-2}$ & $<2.94 \times 10^{-2}$ & $4.80 \times 10^{-1}$ & $1.87 \times 10^{-1}$ & $2.06 \times 10^{-1}$ & $1.96 \times 10^{-8}$ \\
\hline 2 & $1.22 \times 10^{-5}$ & $1.33 \times 10^{-5}$ & $2.64 \times 10^{-4}$ & $<3.97 \times 10^{-6}$ & $4.10 \times 10^{-6}$ & $1.21 \times 10^{-2}$ & $3.36 \times 10^{-3}$ & $6.27 \times 10^{-3}$ & $<2.53 \times 10^{-3}$ & $1.13 \times 10^{-2}$ & $1.61 \times 10^{-9}$ \\
\hline 3 & $<7.30 \times 10^{-5}$ & $1.91 \times 10^{-3}$ & $9.07 \times 10^{-3}$ & $4.05 \times 10^{-4}$ & $1.12 \times 10^{-4}$ & $<6.74 \times 10^{-2}$ & $5.34 \times 10^{-1}$ & $5.21 \times 10^{-1}$ & $2.56 \times 10^{-1}$ & $2.20 \times 10^{-1}$ & $1.42 \times 10^{-8}$ \\
\hline 4 & $<3.76 \times 10^{-6}$ & $<1.16 \times 10^{-5}$ & $3.45 \times 10^{-5}$ & $2.00 \times 10^{-5}$ & $2.74 \times 10^{-6}$ & $<3.91 \times 10^{-3}$ & $<2.93 \times 10^{-3}$ & $4.93 \times 10^{-4}$ & $1.10 \times 10^{-2}$ & $7.83 \times 10^{-3}$ & $1.17 \times 10^{-9}$ \\
\hline 5 & $<4.17 \times 10^{-6}$ & $<9.75 \times 10^{-6}$ & $2.50 \times 10^{-4}$ & $6.51 \times 10^{-5}$ & $1.60 \times 10^{-5}$ & $<4.32 \times 10^{-3}$ & $<2.45 \times 10^{-3}$ & $5.84 \times 10^{-3}$ & $3.63 \times 10^{-2}$ & $3.84 \times 10^{-2}$ & $7.55 \times 10^{-9}$ \\
\hline 6 & $\ldots$ & $\ldots$ & $\ldots$ & $\ldots$ & $<2.76 \times 10^{-5}$ & $\ldots$ & $\ldots$ & $\ldots$ & $\ldots$ & $<6.27 \times 10^{-2}$ & $\ldots$ \\
\hline 7 & $\ldots$ & $\ldots$ & $\ldots$ & $\ldots$ & $<3.11 \times 10^{-5}$ & $\ldots$ & $\ldots$ & $\ldots$ & $\ldots$ & $<6.97 \times 10^{-2}$ & $\ldots$ \\
\hline 8 & $<2.40 \times 10^{-3}$ & $<1.60 \times 10^{-3}$ & $1.69 \times 10^{-2}$ & $1.07 \times 10^{-3}$ & $5.25 \times 10^{-4}$ & $<1.93$ & $<4.45 \times 10^{-1}$ & 1.14 & $8.11 \times 10^{-1}$ & $8.88 \times 10^{-1}$ & $5.98 \times 10^{-8}$ \\
\hline 9 & $\ldots$ & $\ldots$ & $\ldots$ & $\ldots$ & $1.09 \times 10^{-4}$ & $\ldots$ & $\ldots$ & $\ldots$ & $\ldots$ & $2.15 \times 10^{-1}$ & $\ldots$ \\
\hline 10 & $\ldots$ & $\ldots$ & $\ldots$ & $\ldots$ & $4.45 \times 10^{-5}$ & $\ldots$ & $\ldots$ & $\ldots$ & $\ldots$ & $9.62 \times 10^{-2}$ & $7.72 \times 10^{-9}$ \\
\hline 11 & $\ldots$ & $\ldots$ & $\ldots$ & $4.51 \times 10^{-5}$ & $3.33 \times 10^{-5}$ & $\ldots$ & $\ldots$ & $\ldots$ & $4.06 \times 10^{-2}$ & $7.42 \times 10^{-2}$ & $7.17 \times 10^{-9}$ \\
\hline 12 & $4.85 \times 10^{-3}$ & $1.52 \times 10^{-3}$ & $1.10 \times 10^{-2}$ & $\ldots$ & $6.35 \times 10^{-5}$ & 3.79 & $4.21 \times 10^{-1}$ & $6.63 \times 10^{-1}$ & $\ldots$ & $1.33 \times 10^{-1}$ & $1.41 \times 10^{-8}$ \\
\hline 13 & $3.57 \times 10^{-4}$ & $3.05 \times 10^{-4}$ & $2.44 \times 10^{-2}$ & $5.05 \times 10^{-4}$ & $4.91 \times 10^{-4}$ & $3.09 \times 10^{-1}$ & $8.22 \times 10^{-2}$ & 1.79 & $3.69 \times 10^{-1}$ & $8.36 \times 10^{-1}$ & $1.67 \times 10^{-7}$ \\
\hline 14 & $\ldots$ & $\ldots$ & $\ldots$ & $\ldots$ & $1.24 \times 10^{-4}$ & $\ldots$ & $\ldots$ & $\ldots$ & $\ldots$ & $2.42 \times 10^{-1}$ & $\ldots$ \\
\hline 15 & $\ldots$ & $\ldots$ & $\ldots$ & $\ldots$ & $<4.56 \times 10^{-6}$ & $\ldots$ & $\ldots$ & $\ldots$ & $\ldots$ & $<1.24 \times 10^{-2}$ & $\ldots$ \\
\hline 16 & $1.68 \times 10^{-4}$ & $4.18 \times 10^{-5}$ & $2.76 \times 10^{-3}$ & $4.63 \times 10^{-5}$ & $3.94 \times 10^{-5}$ & $1.50 \times 10^{-1}$ & $1.08 \times 10^{-2}$ & $1.18 \times 10^{-1}$ & $3.46 \times 10^{-2}$ & $8.62 \times 10^{-2}$ & $7.13 \times 10^{-9}$ \\
\hline 17 & $6.75 \times 10^{-5}$ & $<5.66 \times 10^{-5}$ & $6.87 \times 10^{-4}$ & $1.98 \times 10^{-5}$ & $5.93 \times 10^{-5}$ & $6.26 \times 10^{-2}$ & $<1.47 \times 10^{-2}$ & $2.07 \times 10^{-2}$ & $2.45 \times 10^{-2}$ & $1.25 \times 10^{-1}$ & $1.58 \times 10^{-8}$ \\
\hline 18 & $\ldots$ & $\ldots$ & $\ldots$ & $1.06 \times 10^{-4}$ & $1.63 \times 10^{-4}$ & $\ldots$ & $\ldots$ & $\ldots$ & $2.77 \times 10^{-1}$ & $3.10 \times 10^{-1}$ & $4.74 \times 10^{-8}$ \\
\hline 19 & $\ldots$ & $\ldots$ & $\ldots$ & $<2.60 \times 10^{-5}$ & $7.67 \times 10^{-5}$ & $\ldots$ & $\ldots$ & $\ldots$ & $<1.35 \times 10^{-1}$ & $1.57 \times 10^{-1}$ & $\ldots$ \\
\hline 20 & $<9.56 \times 10^{-6}$ & $4.07 \times 10^{-5}$ & $8.14 \times 10^{-4}$ & $1.67 \times 10^{-5}$ & $2.53 \times 10^{-5}$ & $<9.58 \times 10^{-3}$ & $1.05 \times 10^{-2}$ & $2.56 \times 10^{-2}$ & $1.28 \times 10^{-2}$ & $5.78 \times 10^{-2}$ & $7.71 \times 10^{-9}$ \\
\hline 21 & $1.42 \times 10^{-4}$ & $8.82 \times 10^{-4}$ & $1.42 \times 10^{-2}$ & $1.09 \times 10^{-4}$ & $7.54 \times 10^{-5}$ & $1.27 \times 10^{-1}$ & $2.42 \times 10^{-1}$ & $9.10 \times 10^{-1}$ & $1.69 \times 10^{-1}$ & $1.55 \times 10^{-1}$ & $2.36 \times 10^{-8}$ \\
\hline 22 & $\ldots$ & $\ldots$ & $\ldots$ & $7.06 \times 10^{-4}$ & $5.15 \times 10^{-4}$ & $\ldots$ & $\ldots$ & $\ldots$ & $7.69 \times 10^{-1}$ & $8.73 \times 10^{-1}$ & $1.42 \times 10^{-7}$ \\
\hline 23 & $8.02 \times 10^{-5}$ & $5.12 \times 10^{-5}$ & $4.89 \times 10^{-3}$ & $1.55 \times 10^{-5}$ & $5.70 \times 10^{-5}$ & $7.38 \times 10^{-2}$ & $1.33 \times 10^{-2}$ & $2.41 \times 10^{-1}$ & $1.50 \times 10^{-2}$ & $1.20 \times 10^{-1}$ & $1.63 \times 10^{-8}$ \\
\hline 24 & $\ldots$ & $\ldots$ & $\ldots$ & $\ldots$ & $9.98 \times 10^{-5}$ & $\ldots$ & $\ldots$ & $\ldots$ & $\ldots$ & $1.99 \times 10^{-1}$ & $\ldots$ \\
\hline 25 & $<2.51 \times 10^{-4}$ & $<1.11 \times 10^{-4}$ & $4.00 \times 10^{-4}$ & $1.56 \times 10^{-5}$ & $<3.96 \times 10^{-5}$ & $<2.21 \times 10^{-1}$ & $<2.92 \times 10^{-2}$ & $1.05 \times 10^{-2}$ & $2.15 \times 10^{-2}$ & $<8.67 \times 10^{-2}$ & $<7.91 \times 10^{-9}$ \\
\hline 26 & $<2.87 \times 10^{-4}$ & $<1.26 \times 10^{-4}$ & $6.15 \times 10^{-4}$ & $<5.51 \times 10^{-5}$ & $5.87 \times 10^{-5}$ & $<2.51 \times 10^{-1}$ & $<3.33 \times 10^{-2}$ & $1.80 \times 10^{-2}$ & $<7.02 \times 10^{-2}$ & $1.23 \times 10^{-1}$ & $1.04 \times 10^{-8}$ \\
\hline 27 & $2.96 \times 10^{-5}$ & $1.26 \times 10^{-4}$ & $2.84 \times 10^{-3}$ & $2.67 \times 10^{-5}$ & $5.13 \times 10^{-5}$ & $2.83 \times 10^{-2}$ & $3.33 \times 10^{-2}$ & $1.22 \times 10^{-1}$ & $2.26 \times 10^{-2}$ & $1.09 \times 10^{-1}$ & $1.41 \times 10^{-8}$ \\
\hline 28 & $\ldots$ & $\ldots$ & $\ldots$ & $\ldots$ & $9.89 \times 10^{-6}$ & $\ldots$ & $\ldots$ & $\ldots$ & $\ldots$ & $2.49 \times 10^{-2}$ & $4.09 \times 10^{-9}$ \\
\hline 29 & $7.18 \times 10^{-5}$ & $<1.76 \times 10^{-5}$ & $1.54 \times 10^{-3}$ & $1.20 \times 10^{-4}$ & $6.10 \times 10^{-5}$ & $6.64 \times 10^{-2}$ & $<4.46 \times 10^{-3}$ & $5.69 \times 10^{-2}$ & $6.87 \times 10^{-2}$ & $1.28 \times 10^{-1}$ & $1.55 \times 10^{-8}$ \\
\hline 30 & $<1.58 \times 10^{-5}$ & $1.18 \times 10^{-4}$ & $2.48 \times 10^{-3}$ & $2.10 \times 10^{-4}$ & $7.61 \times 10^{-5}$ & $<1.55 \times 10^{-2}$ & $3.11 \times 10^{-2}$ & $1.03 \times 10^{-1}$ & $1.55 \times 10^{-1}$ & $1.56 \times 10^{-1}$ & $1.90 \times 10^{-8}$ \\
\hline
\end{tabular}

Notes. Upper limits are provided in case of non-detections. The shown mass accretion rates are computed from the the Br $\gamma$ accretion luminosity, using the available stellar parameters given in Table 1.

Table 6. Line luminosities and derived accretion luminosities for Cha II objects (see caption of Table 5).

\begin{tabular}{|c|c|c|c|c|c|c|c|c|c|c|c|}
\hline $\begin{array}{l}\text { Id } \\
\text { Cha II }\end{array}$ & $\begin{array}{c}L_{[\mathrm{O}] \mathrm{I}} \\
L_{\odot} \\
\end{array}$ & $\begin{array}{c}L_{\text {Ca II }} \\
L_{\odot} \\
\end{array}$ & $\begin{array}{c}L_{\mathrm{H} \alpha} \\
L_{\odot} \\
\end{array}$ & $\begin{array}{c}L_{\mathrm{Pa} \beta} \\
L_{\odot} \\
\end{array}$ & $\begin{array}{c}L_{\mathrm{Br} \gamma} \\
L_{\odot} \\
\end{array}$ & $\begin{array}{c}L_{\mathrm{acc}[\mathrm{O}] \mathrm{I}} \\
L_{\odot} \\
\end{array}$ & $\begin{array}{c}L_{\text {accCa II }} \\
L_{\odot} \\
\end{array}$ & $\begin{array}{c}L_{\mathrm{accH} \alpha} \\
L_{\odot}\end{array}$ & $\begin{array}{c}L_{\mathrm{accPa} \beta} \\
L_{\odot} \\
\end{array}$ & $\begin{array}{c}L_{\mathrm{accB}} \mathrm{Br} \gamma \\
L_{\odot}\end{array}$ & $\begin{array}{l}\dot{M}_{\mathrm{accBr} \gamma} \\
M_{\odot} \mathrm{yr}^{-1}\end{array}$ \\
\hline $1^{a}$ & $1.54 \times 10^{-1}$ & $9.51 \times 10^{-2}$ & $6.34 \times 10^{-1}$ & $1.81 \times 10^{-3}$ & $6.12 \times 10^{-3}$ & $1.05 \times 10^{2}$ & $2.87 \times 10^{1}$ & $1.05 \times 10^{2}$ & 6.58 & 8.10 & $4.49 \times 10^{-7}$ \\
\hline 2 & $<1.89 \times 10^{-4}$ & $<2.39 \times 10^{-4}$ & $4.77 \times 10^{-4}$ & $<9.19 \times 10^{-5}$ & $7.53 \times 10^{-5}$ & $<1.68 \times 10^{-1}$ & $<6.39 \times 10^{-2}$ & $1.31 \times 10^{-2}$ & $<7.86 \times 10^{-2}$ & $1.55 \times 10^{-1}$ & $1.60 \times 10^{-8}$ \\
\hline 3 & $\ldots$ & $\ldots$ & $\ldots$ & $\ldots$ & $1.92 \times 10^{-3}$ & $\ldots$ & $\ldots$ & $\ldots$ & $\ldots$ & 2.85 & $9.10 \times 10^{-7}$ \\
\hline 4 & $\ldots$ & $\ldots$ & $7.87 \times 10^{-4}$ & $8.35 \times 10^{-5}$ & $2.11 \times 10^{-5}$ & $\begin{array}{l}\cdots \\
\ldots\end{array}$ & $\begin{array}{l}\cdots \\
\ldots\end{array}$ & $2.46 \times 10^{-2}$ & $5.90 \times 10^{-2}$ & $4.91 \times 10^{-2}$ & $2.70 \times 10^{-9}$ \\
\hline 5 & $<4.25 \times 10^{-5}$ & $<4.41 \times 10^{-5}$ & $9.04 \times 10^{-5}$ & $1.50 \times 10^{-5}$ & $4.12 \times 10^{-5}$ & $<4.01 \times 10^{-2}$ & $<1.14 \times 10^{-2}$ & $1.64 \times 10^{-3}$ & $1.33 \times 10^{-2}$ & $8.99 \times 10^{-2}$ & $6.42 \times 10^{-9}$ \\
\hline 6 & $<1.27 \times 10^{-4}$ & $2.91 \times 10^{-4}$ & $9.65 \times 10^{-3}$ & $2.95 \times 10^{-5}$ & $1.11 \times 10^{-4}$ & $<1.15 \times 10^{-1}$ & $7.83 \times 10^{-2}$ & $5.63 \times 10^{-1}$ & $2.62 \times 10^{-2}$ & $2.19 \times 10^{-1}$ & $5.42 \times 10^{-8}$ \\
\hline 7 & $<5.60 \times 10^{-5}$ & $7.44 \times 10^{-5}$ & $2.67 \times 10^{-3}$ & $<5.94 \times 10^{-5}$ & $4.85 \times 10^{-5}$ & $<5.23 \times 10^{-2}$ & $1.95 \times 10^{-2}$ & $1.13 \times 10^{-1}$ & $<3.64 \times 10^{-2}$ & $1.04 \times 10^{-1}$ & $6.45 \times 10^{-9}$ \\
\hline 8 & $<1.84 \times 10^{-5}$ & $<1.67 \times 10^{-5}$ & $6.24 \times 10^{-4}$ & $3.18 \times 10^{-5}$ & $1.93 \times 10^{-5}$ & $<1.80 \times 10^{-2}$ & $<4.23 \times 10^{-3}$ & $1.84 \times 10^{-2}$ & $1.64 \times 10^{-2}$ & $4.53 \times 10^{-2}$ & $4.50 \times 10^{-9}$ \\
\hline 9 & $<3.84 \times 10^{-5}$ & $<8.19 \times 10^{-5}$ & $3.38 \times 10^{-4}$ & $3.20 \times 10^{-5}$ & $4.82 \times 10^{-5}$ & $<3.64 \times 10^{-2}$ & $<2.14 \times 10^{-2}$ & $8.55 \times 10^{-3}$ & $1.83 \times 10^{-2}$ & $1.03 \times 10^{-1}$ & $4.73 \times 10^{-9}$ \\
\hline 10 & $<8.49 \times 10^{-5}$ & $<1.32 \times 10^{-4}$ & $5.58 \times 10^{-4}$ & $<5.63 \times 10^{-5}$ & $5.48 \times 10^{-5}$ & $<7.80 \times 10^{-2}$ & $<3.49 \times 10^{-2}$ & $1.60 \times 10^{-2}$ & $<4.28 \times 10^{-2}$ & $1.16 \times 10^{-1}$ & $1.10 \times 10^{-8}$ \\
\hline 11 & $1.35 \times 10^{-4}$ & $1.01 \times 10^{-4}$ & $2.50 \times 10^{-3}$ & $5.97 \times 10^{-5}$ & $2.78 \times 10^{-5}$ & $1.22 \times 10^{-1}$ & $2.65 \times 10^{-2}$ & $1.04 \times 10^{-1}$ & $5.33 \times 10^{-2}$ & $6.31 \times 10^{-2}$ & $4.68 \times 10^{-9}$ \\
\hline 12 & $2.83 \times 10^{-5}$ & $<5.54 \times 10^{-5}$ & $5.92 \times 10^{-4}$ & $2.74 \times 10^{-5}$ & $1.22 \times 10^{-5}$ & $2.72 \times 10^{-2}$ & $<1.44 \times 10^{-2}$ & $1.72 \times 10^{-2}$ & $2.19 \times 10^{-2}$ & $3.01 \times 10^{-2}$ & $7.16 \times 10^{-9}$ \\
\hline 13 & $4.75 \times 10^{-5}$ & $<1.14 \times 10^{-4}$ & $8.56 \times 10^{-4}$ & $<5.22 \times 10^{-5}$ & $2.68 \times 10^{-5}$ & $4.46 \times 10^{-2}$ & $<3.01 \times 10^{-2}$ & $2.73 \times 10^{-2}$ & $<4.19 \times 10^{-2}$ & $6.11 \times 10^{-2}$ & $3.60 \times 10^{-8}$ \\
\hline 14 & $<6.05 \times 10^{-5}$ & $2.47 \times 10^{-4}$ & $3.33 \times 10^{-3}$ & $4.01 \times 10^{-5}$ & $4.38 \times 10^{-5}$ & $<5.63 \times 10^{-2}$ & $6.63 \times 10^{-2}$ & $1.49 \times 10^{-1}$ & $3.65 \times 10^{-2}$ & $9.49 \times 10^{-2}$ & $4.53 \times 10^{-9}$ \\
\hline 15 & $1.66 \times 10^{-4}$ & $2.96 \times 10^{-4}$ & $1.48 \times 10^{-2}$ & $4.55 \times 10^{-4}$ & $2.02 \times 10^{-4}$ & $1.48 \times 10^{-1}$ & $7.95 \times 10^{-2}$ & $9.63 \times 10^{-1}$ & $3.92 \times 10^{-1}$ & $3.76 \times 10^{-1}$ & $2.01 \times 10^{-8}$ \\
\hline 16 & $<3.21 \times 10^{-5}$ & $<3.63 \times 10^{-5}$ & $8.89 \times 10^{-5}$ & $3.66 \times 10^{-5}$ & $2.38 \times 10^{-5}$ & $<3.06 \times 10^{-2}$ & $<9.36 \times 10^{-3}$ & $1.61 \times 10^{-3}$ & $2.52 \times 10^{-2}$ & $5.49 \times 10^{-2}$ & $3.02 \times 10^{-9}$ \\
\hline 17 & $1.17 \times 10^{-5}$ & $2.21 \times 10^{-5}$ & $4.63 \times 10^{-4}$ & $<7.43 \times 10^{-5}$ & $<2.49 \times 10^{-5}$ & $1.16 \times 10^{-2}$ & $5.65 \times 10^{-3}$ & $1.27 \times 10^{-2}$ & $<3.78 \times 10^{-2}$ & $<5.71 \times 10^{-2}$ & $<5.49 \times 10^{-9}$ \\
\hline
\end{tabular}

Notes. The mass accretion rates are computed from the the $\mathrm{Br} \gamma$ accretion luminosity, using the available stellar parameters given in Table 2. ${ }^{(a)} \mathrm{A}$ more detailed analysis of ChaII-1 (DK Cha) is presented in García López et al. (2011).

not observed such a tight correlation between $L_{\text {acc }}$ and $L_{*}$ as the one we get with Br $\gamma$ : Garcia Lopez et al. (2006) have used Br $\gamma$ to derive $L_{\text {acc }}$ in a sample of Herbig stars and have observed a dispersion of about two dex; Natta et al. (2006) found a scatter of about two orders of magnitude using $\mathrm{Pa} \beta$ to compute $L_{\text {acc }}$ in their low-mass Oph sample, which is similar to the $L_{\text {acc }}(\mathrm{Pa} \beta)$ dispersion we see in our data.
Although in general we cannot expect young sources with similar $L_{*}$ to present comparable accretion luminosities, in this work we are analysing a homogeneous sample of objects of the same star-forming cloud that are probably in a similar evolutionary stage (almost all sources are Class IIs). Therefore, it seems totally reasonable to expect that targets with similar $L_{*}$ will likely display $L_{\mathrm{acc}}$ of the same order of magnitude. In this sense, therefore, the $\operatorname{Br} \gamma$ appears to be the most reliable tracer, 


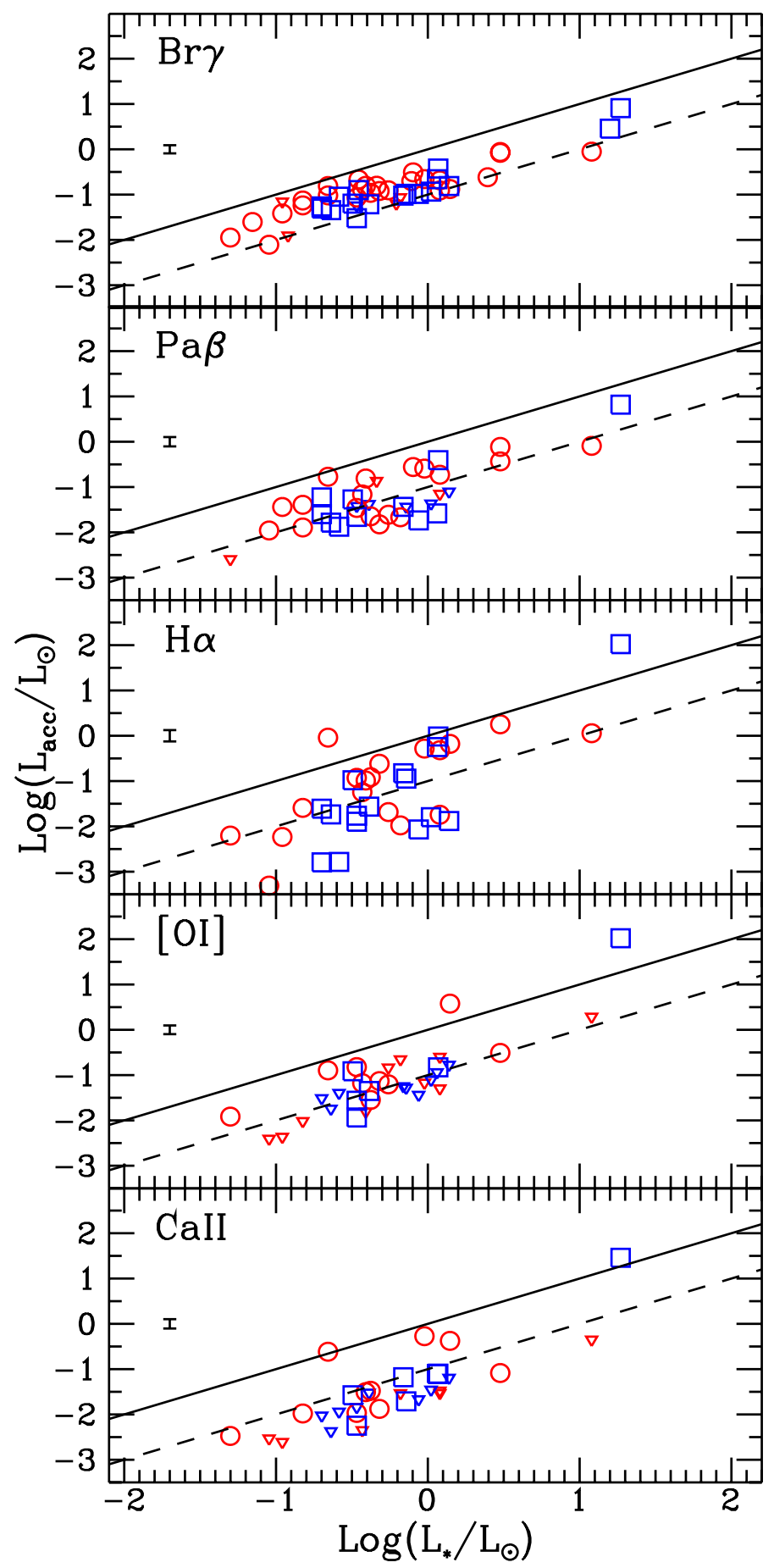

Fig. 3. $L_{\text {acc }}$ values for the different accretion tracers considered (see Tables 5 and 6) plotted as a function of $L_{*}$ : from top to bottom $\operatorname{Br} \gamma$, $\mathrm{Pa} \beta, \mathrm{H} \alpha,\left[\mathrm{O}_{\text {I }}\right] \lambda 6300, \mathrm{Ca}$ II $\lambda 8542$. Red circles and blue squares refer to detections of Cha I and Cha II objects, respectively. Upper limits are indicated by downward triangles. The solid and dashed lines show the locus where $L_{\text {acc }}=L_{*}$ and $L_{\text {acc }}=0.1 L_{*}$, respectively. The error bars reflect only the assumed uncertainty of 0.5 mag for the 2MASS $K$ band magnitude used as reference for the absolute flux calibration of the spectra (see Sect. 4).

because it provides the smallest scatter of $L_{\mathrm{acc}}$ values throughout the covered range of $L_{*}$. In addition, the values of $L_{\text {acc }}$ we derive from the $\operatorname{Br} \gamma$ relationship, which are of the order of a fraction of the stellar luminosity $\left(\sim 0.1-1 L_{*}\right)$, agree with what may be expected for typical Class II objects that are, by definition, characterised by a bolometric luminosity dominated by the photospheric contribution.

We point out that the infrared tracers are also less affected by reddening, so any error on the extinction value should produce a smaller effect on the $L_{\text {acc }}$ derived from $\mathrm{Br} \gamma$ and $\mathrm{Pa} \beta$. Indeed, the different observed scatters might be caused in principle (at least in part) by a wrong extinction estimate. However, considering the fairly low values of $A_{V}$ characterising most of our sources (see Tables 1 and 2), even $100 \%$ relative errors on this parameter are not sufficient to explain the very large scatters observed, especially for the optical lines.

As already mentioned, $\mathrm{Br} \gamma$ is in general expected to be emitted in much more compact regions around the source with respect to the other tracers. Additionally, the fact that at least part of the line emission is indeed coming from infalling material (hence from a region very close to the source) has often been shown by the analysis of the Br $\gamma$ profiles (e.g. Folha \& Emerson 2001). Thus, flux losses due to the signal actually falling within the slit are less important for $\mathrm{Br} \gamma$, even if part of the emission comes from a wind/jet. The smaller dispersion we observe is likely linked to this effect as well.

Based on the previous considerations, we therefore chose to adopt the accretion luminosities derived from $\mathrm{Br} \gamma$.

The larger scatters observed for the other tracers may probably be ascribed to the different contributions to the emission lines, which may vary between our sources and those of the sample where the relationships were calibrated, for instance because of different line excitation mechanisms and/or different line formation environments. An important piece of evidence in favour of this scenario is that the other tracers provide mean accretion luminosities that are different from those obtained through $\operatorname{Br} \gamma$, in particular for some ranges of $L_{*}$ values. To better analyse these discrepancies, we show in Fig. 4 direct comparisons between the accretion luminosities derived from $\mathrm{Br} \gamma$ and those inferred from the other lines. We notice no particular difference between the distribution of Cha I and Cha II objects in these plots and can consequently rule out effects related to specific properties of one of the two clouds.

There is evidence for $\operatorname{Br} \gamma$ and $\operatorname{Pa} \beta$ that in many cases $\operatorname{Pa} \beta$ underestimates the accretion luminosity with respect to $\mathrm{Br} \gamma$ in sources with $-2<\log L_{\text {acc }}(\operatorname{Pa} \beta)<-1$, which corresponds to a range $0.1-1 L_{\odot}$ for $L_{*}$. In Sect. 8 we show that these sources are those displaying unusually low values of the $\mathrm{Pa} \beta / \mathrm{Br} \gamma$ ratio (see Fig. 7). In particular, these ratios are lower than those typically observed in the samples of T Tauri stars over which the $\mathrm{H}$ I relationships were calibrated. Indeed, if we exclude these objects (indicated by filled symbols in Fig. 4) the $\mathrm{Br} \gamma$ and $\mathrm{Pa} \beta$ accretion luminosity determinations appear to agree very well. Conversely, the low- $\mathrm{Pa} \beta / \mathrm{Br} \gamma$ objects seem to be randomly distributed in the plots that show the comparison with the other tracers.

$L_{\text {acc }}$ values from $\mathrm{Br} \gamma$ and $\mathrm{H} \alpha$ are instead very different. Indeed, the $\mathrm{H} \alpha$ accretion luminosities seem to follow a different trend than $\mathrm{Br} \gamma$ : they appear underestimated for $L_{\mathrm{acc}}(\mathrm{Br} \gamma) \lesssim 0.1 L_{\odot}$ and overestimated for $L_{\mathrm{acc}}(\mathrm{Br} \gamma) \gtrsim 0.1 L_{\odot}$. This effect is likely associated with the presence of several contributions to the line: for example enhanced chromospheric emission (e.g. Hamann \& Persson 1992) or emission/absorption from outflowing material, the presence of which is often testified by P Cygni profiles (e.g. Reipurth et al. 1996; Calvet et al. 1992). Also the contribution from direct photoionisation by stellar photons might become an important factor at higher luminosities. In all these scenarios, part of the $\mathrm{H} \alpha$ emission acts as an indirect tracer of the accretion process, and might be affected 
S. Antoniucci et al.: POISSON observations: emission lines as accretion tracers in YSOs
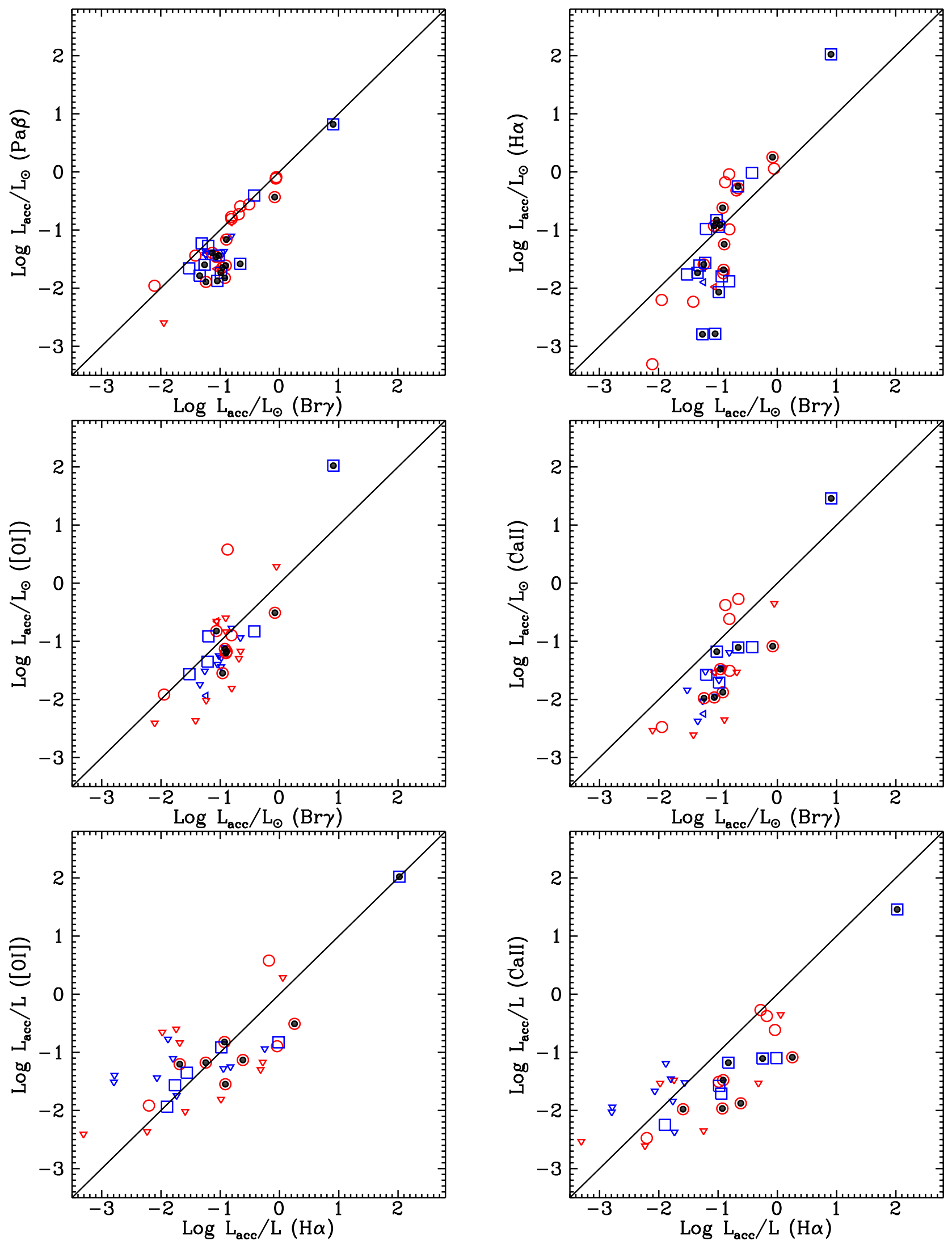

Fig. 4. Comparison between $L_{\mathrm{acc}}(\mathrm{Br} \gamma)$ and $L_{\text {acc }}$ determinations from the other four tracers and between $L_{\text {acc }}(\mathrm{H} \alpha)$ and $L_{\text {acc }}$ from [O I] and Ca II. The red circles and blue squares refer to Cha I and Cha II objects, respectively, whereas the triangles of the same colours indicate upper limits. The dashed lines mark the locus of equal accretion luminosity. The symbols filled with a black dot identify the sources displaying low values of the $\operatorname{Pa} \beta / \operatorname{Br} \gamma$ ratio (see Sect. 8 and Fig. 7). 
by strong source-to-source variations. For instance, if there is a significant contribution from a wind, then this emission is probably spatially extended (much more than $\operatorname{Br} \gamma$ ), so that flux losses might become important, especially when using empirical relationships calibrated on the basis of spectroscopic observations carried out with different conditions and instrument configurations. This would explain in part the bigger scatter in $L_{\text {acc }}$ values obtained from $\mathrm{H} \alpha$.

We expect a similar situation for [O I ] as well, because forbidden oxygen emission is typically associated with outflowing winds/jets from protostars (e.g. Ray et al. 2007). Indeed, if we take into account the upper limits on $L_{\mathrm{acc}}\left(\left[\mathrm{O}_{\mathrm{I}}\right]\right)$, we note that the shape of the correlation between $L_{\text {acc }}$ values from $\mathrm{Br} \gamma$ and [O I] looks similar to the one between $\mathrm{Br} \gamma$ and $\mathrm{H} \alpha$. A direct comparison between accretion luminosities derived from $\mathrm{H} \alpha$ and [O I] shows that these tracers provide on average roughly consistent results, although the $L_{\mathrm{acc}}(\mathrm{H} \alpha)-L_{\mathrm{acc}}([\mathrm{O} \mathrm{I}])$ correlation is characterised by a large scatter around the locus of equal accretion luminosity (see Fig. 4). An evident correlation between $L_{\text {acc }}(\mathrm{H} \alpha)$ and [O I] emission was also observed, for example, by Fang et al. (2009) in their sample of Orion YSOs.

Furthermore, the accretion luminosities derived from Ca II seem to present the same trend as [O I ] and $\mathrm{H} \alpha$ when compared to $L_{\mathrm{acc}}(\mathrm{Br} \gamma)$, which might testify to a common origin of these lines from outflowing gas. In particular, they appear to be underestimated with respect to $\mathrm{Br} \gamma$ for $L_{\mathrm{acc}} \lesssim 0.1 L_{\odot}$, which is similar to what is observed for $\mathrm{Pa} \beta$ as well. Interestingly, $L_{\text {acc }}(\mathrm{Ca}$ II) appear to be correlated, but consistently (i.e. over the entire $L_{\text {acc }}$ range) underestimated (about one dex) with respect to those from $\mathrm{H} \alpha$.

In summary, our analysis shows that that the empirical line$L_{\text {acc }}$ relationships, calibrated on the basis of various surveys (but considering almost exclusively Taurus YSOs, see appendix), actually provide systematically different results when applied to our sample. The Br $\gamma$ line gives the smallest dispersion of $L_{\mathrm{acc}}$ over the entire range of $L_{*}$, whereas the other tracers, especially $\mathrm{H} \alpha$, provide much more scattered $L_{\mathrm{acc}}$ results that are not expected for our homogeneous sample of targets. In this sense, the $\mathrm{Br} \gamma$ relationship is the most reliable, because it looks to be less subject to biases when applied to other YSO samples.

\section{Accretion properties of Cha I and Cha II objects}

In Fig. 5 we analyse the connection between the accretion luminosity $L_{\text {acc }}$ (derived from $\mathrm{Br} \gamma$ ) and other source parameters. The top panel shows the tight correlation of $L_{\text {acc }}$ and $L_{*}$ : all sources have accretion luminosities in the range $0.1 L_{*}-1 L_{*}$, but the two samples seem to display a different mean value of $L_{\text {acc }}$. We find indeed that the formal average accretion to stellar luminosity ratio for the Cha I and Cha II samples is $L_{\text {acc }} / L_{*}=0.29$ and $L_{\text {acc }} / L_{*}=0.21$, respectively.

Because the infrared luminosity $L_{\mathrm{IR}}$ is very similar or comparable to $L_{*}$ for all sources (see Sect. 3), we find that $L_{\text {acc }}(\operatorname{Br} \gamma)$ is also correlated to $L_{\mathrm{IR}}$ (central panel). Conversely, we cannot derive any significant connection between $L_{\text {acc }}$ and the YSO Class, also owing to the relative paucity of Flat and Class I sources. The bottom panel of Fig. 5 shows that the Class II objects dominating the sample actually display a very wide range of accretion luminosities.

Since the $L_{*}$ of an object is determined by its mass and age, it is interesting to analyse how $L_{\text {acc }}$ correlates with these quantities. However, basically all sources of the sample have an estimated age in the range 1-10 Myr (except for three objects in Cha II
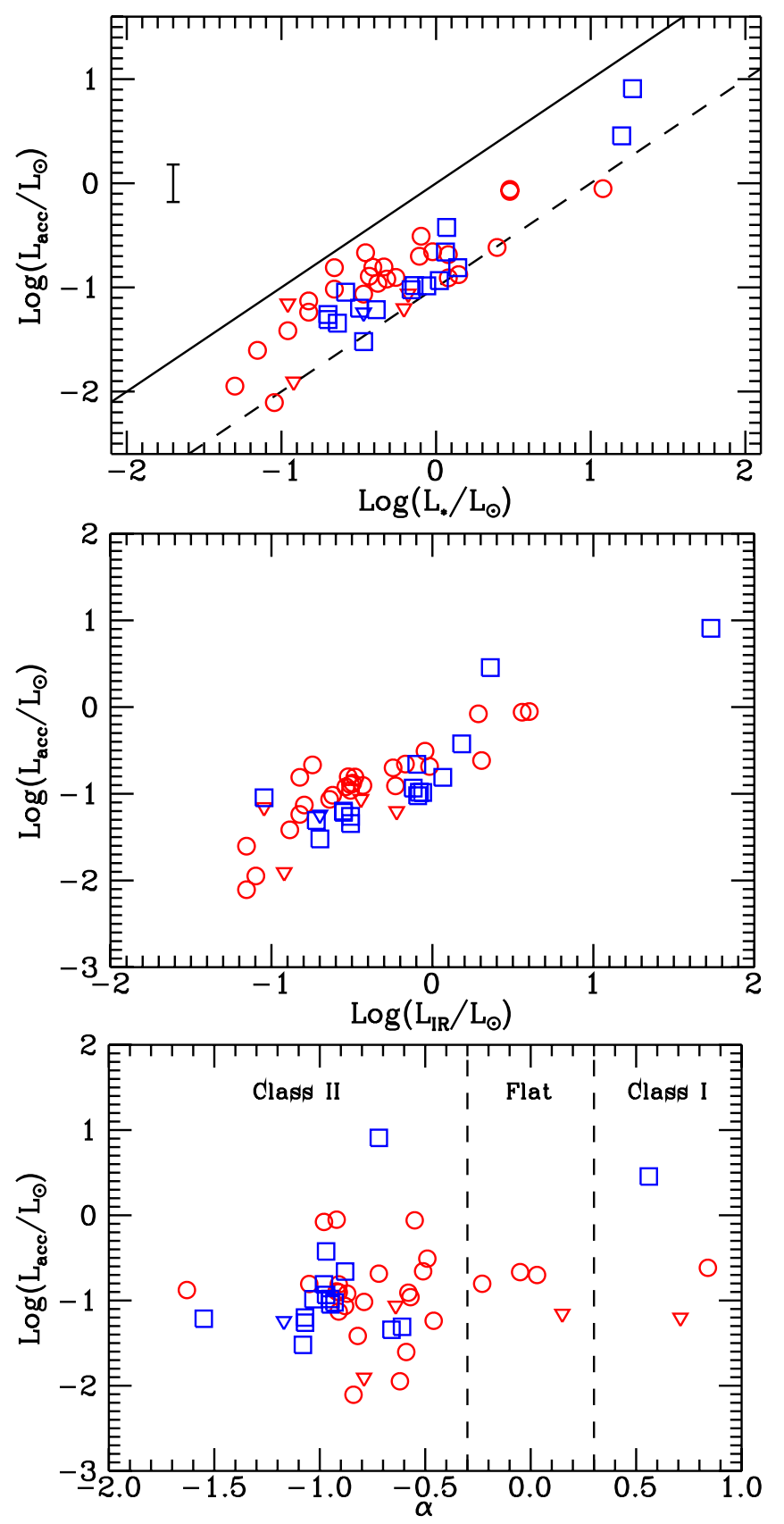

Fig. 5. Accretion luminosity (derived from $\operatorname{Br} \gamma$ ) as a function of $L_{*}$ (top), $L_{\mathrm{IR}}$ (centre), and spectral index $\alpha_{2-24}$ (bottom). Red circles and blue squares refer to Cha I and Cha II sources, respectively, while triangles of the same colours mark upper limits on $L_{\text {acc }}$.

with ages between 10 and $15 \mathrm{Myr}$ ), with a vast majority of objects being less than 5 Myr old (see Luhman 2007; Spezzi et al. 2008), i.e. substantially coeval. Consequently, the very narrow range of ages covered by the objects does not allow us to analyse the relation between accretion luminosity and age properly. The $L_{\text {acc }}-L_{*}$ correlation that we observe should therefore be ascribed mostly to a dependence of the accretion process on the stellar mass. This $L_{\mathrm{acc}}-M_{*}$ correlation is shown in the top panel of Fig. 6.

From $L_{\text {acc }}(\mathrm{Br} \gamma)$ we computed the mass accretion rates $\dot{M}_{\text {acc }}$ using (e.g. Gullbring et al. 1998):

$\dot{M}_{\mathrm{acc}}=\frac{L_{\mathrm{acc}} R_{*}}{G M_{*}}\left(1-\frac{R_{*}}{R_{\mathrm{in}}}\right)^{-1}$, 

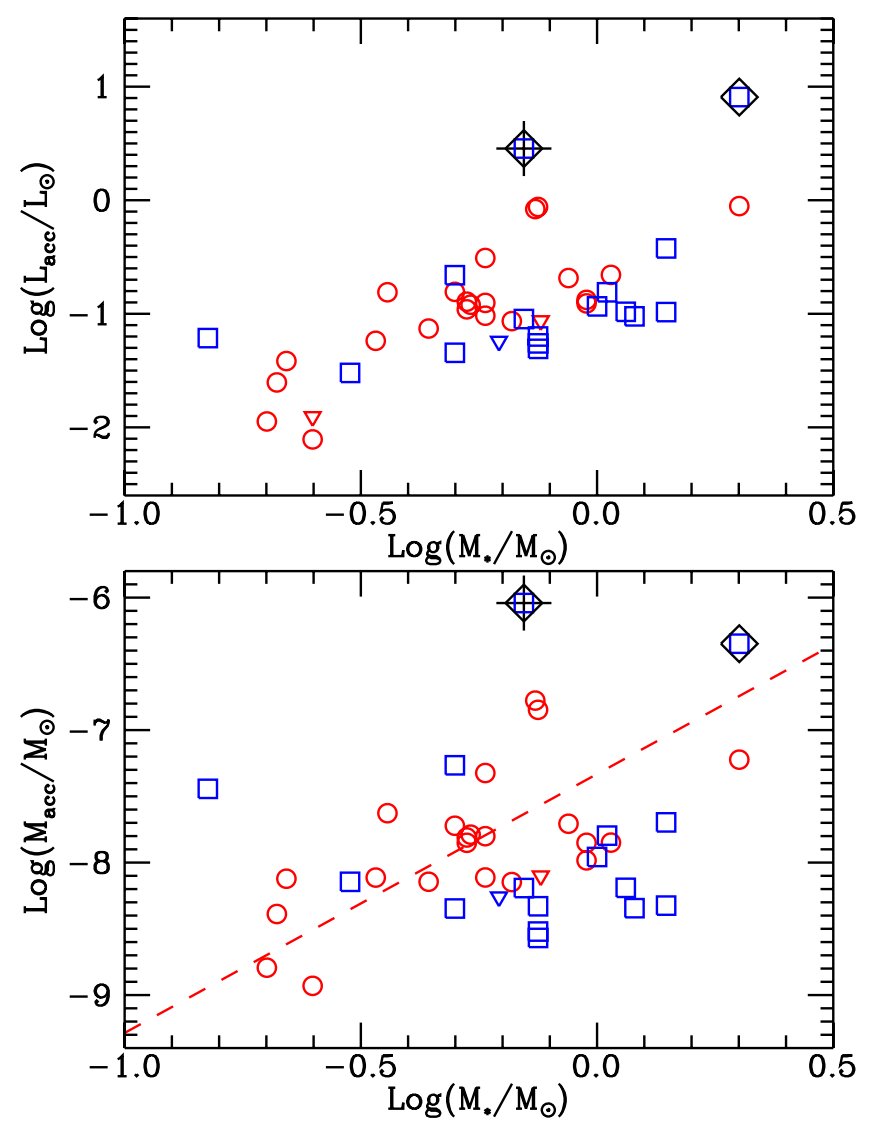

Fig. 6. $L_{\text {acc }}$ (top panel) and $\dot{M}_{\text {acc }}$ (bottom) as a function of the stellar mass $M_{*}$. Red circles and blue squares refer to Cha I and Cha II objects, respectively. Upper limits on $\dot{M}_{\text {acc }}$ are marked by triangles of the same colours. The only Class I object (Cha II-3) is indicated by a cross. Diamonds identify the objects with a highly uncertain mass estimate (Cha II-1 and Cha II-3, see text for details). The dashed red line in the bottom panel shows the best-fit relation $\dot{M}_{\text {acc }} \propto M_{*}^{1.95 \pm 0.34}$ obtained for Cha I objects.

where $M_{*}$ and $R_{*}$ are the known stellar parameters. We assume the factor $1-R_{*} / R_{\text {in }}$ equal to 0.8 , which implies an inner disc radius $R_{\text {in }}=5 R_{*}$. The $\dot{M}_{\text {acc }}$ obtained (see Tables 5 and 6 ) are of the order of $10^{-7}-10^{-9} M_{\odot} \mathrm{yr}^{-1}$, in the range of values observed in many Class II objects (see e.g. Gullbring et al. 1998; Natta et al. 2006; White et al. 2007).

We have compared the derived mass accretion rates with those computed by Spezzi et al. (2008) for Cha II objects from the $\mathrm{H} \alpha 10 \%$-peak line width (Natta et al. 2004). For almost all sources the $\dot{M}_{\text {acc }}$ are lower (even two orders of magnitude in some cases) than the values we infer from $\mathrm{Br} \gamma$. Interestingly, in Fig. 4 (2nd panel) we find that the majority of Cha II sources are located in the region of the plot where $\mathrm{H} \alpha$ accretion luminosities underestimate those from $\operatorname{Br} \gamma$. This finding might also suggest, albeit in a qualitative way, that the $\mathrm{H} \alpha 10 \%$ relation employed by the authors is affected by a trend similar to the one that was shown for the relationship between $\mathrm{H} \alpha$ flux and $L_{\text {acc. }}$.

The computed mass accretion rates depend of course on the mass adopted for the sources. In general, the estimate of the mass will vary depending on the pre-main sequence evolutionary tracks considered. In our case, however, the masses adopted for Cha I and Cha II objects have been determined by Luhman et al. (2008) and Spezzi et al. (2008) using the same evolutionary models of Baraffe et al. (1998), so that this should not introduce a systematic error in the $\dot{M}_{\text {acc }}$ results of the two samples.
Spezzi et al. (2008) have actually investigated in their paper the effect of adopting different evolutionary models, providing also the mass estimates obtained from the models of D'Antona \& Mazzitelli (1997) and Palla \& Stahler (1999). We checked how our results changed by using these different masses for Cha II objects and found that although the mass values may differ even by a factor 2-3 in a few cases, there is no significant change in the general distribution of $\dot{M}_{\text {acc }}$.

The assumed mass estimate of Cha II- 1 and Cha II-3 was obtained from the D'Antona \& Mazzitelli (1997) evolutionary tracks, because Baraffe et al. (1998) models did not provide a clear result for these more luminous sources. Moreover, Spezzi et al. (2008) explicitly state that the stellar parameters provided for Cha II-3 (which is a Class I) must be taken with care.

In the lower panel of Fig. 6 we show $\dot{M}_{\text {acc }}$ as a function of the stellar mass. We remark that all the sources in the diagram are classified as Class IIs (i.e. they have a negative spectral index), except for Cha II-3. The plot shows a general visible trend of $\dot{M}_{\text {acc }}$ increasing with the stellar mass, in agreement with the expected correlation between accretion and $M_{*}$. This is evident in particular for Cha I objects. However, the observed spread of $\dot{M}_{\text {acc }}$ for any value of $M_{*}$, especially in the case Cha II sources, is larger than the corresponding dispersion in the $L_{\mathrm{acc}}-L_{*}$ plot. We point out, however, that the sources displaying the highest mass accretion rates are Cha II- 1 and Cha II-3, for which the result is likely biased because of the problems mentioned in deriving their stellar mass. Indeed, we notice an evident increase of the spread passing from $L_{\text {acc }}$ vs. $L_{*}$ to $L_{\text {acc }}$ vs. $M_{*}$ and then to $\dot{M}_{\text {acc }}$ vs. $M_{*}$ (Figs. 5 and 6), i.e. introducing the stellar mass in the plotted quantities. Hence, this finding suggests that the larger dispersion observed in the $\dot{M}_{\text {acc }}-M_{*}$ plot is most probably the result of the (often large) uncertainties in the determination of the stellar masses.

Adopting a bisection linear regression on Cha I points, we derive a best-fit relationship $\dot{M}_{\text {acc }} \propto M_{*}^{1.95 \pm 0.34}$. This power law index is close to the one found in other low-mass star-forming regions, e.g. 1.8 (Natta et al. 2004, in $\rho$ Oph), 1.87 (Herczeg \& Hillenbrand 2008, in Taurus), 2.1 (Muzerolle et al. 2005, using observations in various clouds), 1.6 (Rigliaco et al. 2011, in $\sigma$ Ori), whereas it significantly differs from the 2.8-3.4 value measured for example in Orion by Fang et al. (2009). Because Cha II points are much more scattered in the plot, it is difficult to clearly identify a similar trend, so that we could not perform a reasonable linear fit. Excluding Cha II-1 and Cha II-3, we see however that the majority of the Cha II objects provide accretion rates lower than in Cha I (for the same value of the stellar mass), in agreement with the observed difference of the average $L_{\text {acc }} / L_{*}$ values of the two clouds. This small difference in the $\dot{M}_{\text {acc }}$ value between Cha I and Cha II, might actually reflect the difference between the mean age of the two associations (i.e. $\sim 1-$ 2 Myr, see Sect. 3), suggesting a decrease of the mean accretion rate with time.

\section{8. $\mathrm{Pa} \beta / \mathrm{Br} \gamma$ ratio}

The analysis of the (extinction-corrected) $\mathrm{Pa} \beta / \mathrm{Br} \gamma$ ratio (hereafter $R$ ) provides important information on the regions and mechanisms responsible for the $\mathrm{H}_{\mathrm{I}}$ emission, because this ratio is sensitive to the temperature and density of the emitting gas. We find that the discrepancies between $L_{\mathrm{acc}}(\mathrm{Br} \gamma)$ and $L_{\mathrm{acc}}(\mathrm{Pa} \beta)$ observed in many objects (see first panel of Fig. 4) can be related to a different intrinsic value of the $\mathrm{Pa} \beta / \mathrm{Br} \gamma$ ratio in these sources with respect to the ratio measured in the sample where the $\mathrm{H}_{\text {I }}$ relationships were calibrated. 
A\&A 534, A32 (2011)
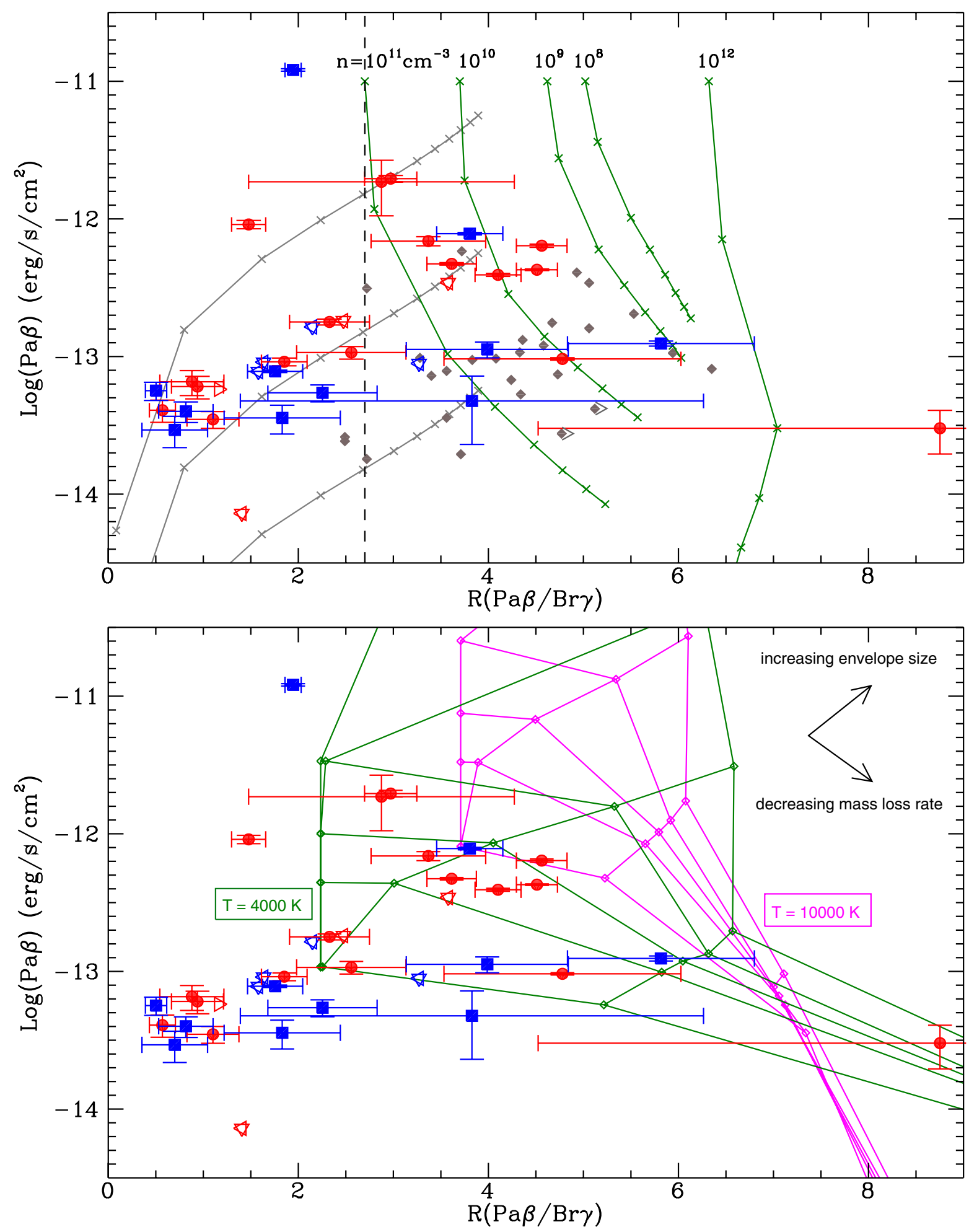

Fig. 7. $\mathrm{Pa} \beta$ flux as a function of the $\mathrm{Pa} \beta / \mathrm{Br} \gamma$ ratio for ChaI (red points) and ChaII (blue points) sources. The open triangles indicate upper/lower limits, depending on their orientation. All quantities are extinction-corrected. A comparison with various emission scenarios is presented (see text for details). (Upper panel) The grey solid lines show the expected emission from gas in an optically thick region in LTE and refer to three different emitting areas $\left(0.2,2,20 \times 10^{22} \mathrm{~cm}^{2}\right)$ and temperatures (crosses) from 1000 to $12000 \mathrm{~K}$ (from left to right in steps of $1000 \mathrm{~K}$ ). The green curves on the right side of the plot show the expected emission in case-B conditions from a gas with density $n$, indicated at the top of each curve, and temperatures (crosses) from 500 to $15000 \mathrm{~K}$ (downwards, steps corresponding to 500, 1000, 3000, 5000, 7500, 10000,12500 , and $15000 \mathrm{~K}$ ). The case B curves have been normalised to obtain the same $\mathrm{Pa} \beta$ emission at $T=500 \mathrm{~K}$. The vertical dashed line at $R \sim 2.7$ marks the lower limit of the region where ratios are still compatible with optically thin emission in case B conditions. For comparison's sake the grey diamonds show the ratios measured in Taurus CTTSs by Muzerolle et al. (1998b). (Lower panel) Results obtained from a model of a spherically symmetric expanding wind in LTE conditions (see Nisini et al. 1995) for gas temperatures of $T=4000 \mathrm{~K}$ (green) and $T=10000 \mathrm{~K}$ (magenta) are overplotted. For each temperature the open diamonds show the expected emission for four different values of the mass loss rate $\left(\dot{M}_{\text {loss }}\right.$ from $10^{-6}$ to $\left.10^{-9} M_{\odot} \mathrm{yr}^{-1}\right)$ and envelope thickness $\left(1,2,5,10\right.$, and $100 R_{*}$ ), these parameters varying in the direction indicated by the arrows. The lines (solid green for $T=4000 \mathrm{~K}$ and dotted-dashed magenta for $T=10000 \mathrm{~K}$ ) connect the results obtained with the same thickness or $\dot{M}_{\text {loss }}$. 
In Fig. 7 we show $R$ as a function of the de-reddened $\mathrm{Pa} \beta$ flux. We notice that the sources of our samples display quite a wide range of $R$ values (from $\sim 0.5$ up to $\sim 9$ ), with no significant distribution difference between Cha I and Cha II objects. Remarkably, the figure also shows that there is a large group of sources $(15$ out of 28$)$ presenting low $(\$ 2.5) R$ values and $\mathrm{Pa} \beta$ intrinsic fluxes of the order of $10^{-14}-10^{-13} \mathrm{erg} \mathrm{s}^{-1} \mathrm{~cm}^{-2}$, which appears to be somewhat separated from the rest of the objects that display intermediate-high ratios.

The position of the sources on the diagram depends of course on the extinction value, so we have analysed the effects of possible errors on the adopted $A_{\mathrm{V}}$, especially in regard to the group of objects showing low ratios. For instance, considering an $A_{\mathrm{V}}$ variation of +4 mag (i.e. adopted extinction is underestimated), we obtain an increase of both the $\mathrm{Pa} \beta$ intrinsic flux (by a factor $\sim 3.4$ ) and $R$ (factor $\sim 2.1$ ). Because most of the sources displaying low ratios have extinction estimates lower than 4 mag, this variation would represent a relative error greater than $100 \%$ on the extinction. In any case, even assuming such a scenario, the sources now displaying $R \lesssim 1$ would remain in the low ratio region of the diagram. Conversely, if the adopted extinction were overestimated, the derived $R$ value would be even lower. At any rate, the quite uniform distribution of both Cha I and Cha II objects in the different regions of the diagram suggests that there is no relevant systematic error between the extinction estimates of the two samples. Additionally, we point out that because $R$ depends only on the goodness of the inter-calibration of the BG and RG segments, it does not suffer from the uncertainty on the absolute flux scale calibration of the spectra, whereas this affects the intrinsic $\operatorname{Pa} \beta$ flux.

Now, we can show that different values of $R$ can be actually associated with different regimes of emission. This can be easily seen in the upper panel of Fig. 7, where we display the expected loci for two standard emission scenarios: optically thick lines from a gas in LTE conditions, i.e. blackbody-like emission, and case-B emission (Hummer \& Storey 1987), i.e. optically thin lines when level populations are mostly determined by radiative cascade from the continuum.

The blackbody curves on the left side of the plot (solid grey lines) were computed assuming a $F W H M$ of $100 \mathrm{~km} \mathrm{~s}^{-1}$ (lines are not resolved in our spectra) and considering different projected areas for the emission region: (bottom to top) $2 \times 10^{21}$, $2 \times 10^{22}$, and $2 \times 10^{23} \mathrm{~cm}^{2}$. To simplify the diagram we have traced these curves assuming a single average value for the distance to the Cha clouds: because the distances are very similar, this does not significantly affect their location on the plot. The crosses along each line refer to different values of the gas temperature increasing from $1000 \mathrm{~K}$ up to 12000 (left to right), in steps of $1000 \mathrm{~K}$. We note that the curves cover a range of $R$ spanning from very low values up to about 4 .

The case- $\mathrm{B}$ curves on the right side of the plot (solid green lines) show the expected emission from a gas with density $n$ (from $10^{8}$ to $10^{12} \mathrm{~cm}^{-3}$ ) within a given emitting volume $V$. The crosses along the curves indicate different values of the gas temperature from $500 \mathrm{~K}$ up to 15000 (top to bottom). The various lines have been normalised so as to obtain the same $\mathrm{Pa} \beta$ emission (which is proportional to the $n^{2} V$ product) at $T=500 \mathrm{~K}$. We note that for case-B conditions the lowest values of $R$ (around 2.7) are obtained for gas densities of the order of $10^{11} \mathrm{~cm}^{-3}$ (and low temperatures), after which $R$ starts to increase with increasing density (see the location of the $10^{12} \mathrm{~cm}^{-3}$ density curve).

Therefore, although the expected $\mathrm{Pa} \beta$ flux may vary in the two cases depending on the assumed emission area or $n^{2} V$ product, evidently low values of $R(\lesssim 2.5)$ are obtainable only for optically thick and thermalised lines for the reasonable range of temperatures explored, whereas high ratios $(\gtrsim 4)$ indicate optically thin lines.

Hence, the low $R$ values observed in many objects provide evidence that the $\operatorname{Pa} \beta$ and $\operatorname{Br} \gamma$ lines originate in an LTE gas at temperatures $1000 \mathrm{~K}<T \lesssim 4000-5000 \mathrm{~K}$. In this scenario, the size of the emitting (projected) area can be estimated directly from the position of the points with respect to the blackbody curves. The majority of the objects is clearly located in the region between the $2 \times 10^{22} \mathrm{~cm}^{2}$ and $2 \times 10^{23} \mathrm{~cm}^{2}$ curves or around them. This indicates an emitting region that is about $0.6-6$ times the projected area of a young star with a typical radius of $2 R_{\odot}$.

In addition to the standard emission scenarios considered above, we have also taken into account the case of emission from a spherically symmetric expanding wind in LTE conditions. In the simple model we employed (see Nisini et al. 1995, for a comprehensive description), we assume a fixed gas temperature, an envelope inner radius $r_{i}=1 R_{*}$, and a radial velocity law $\left(v \propto\left(1-r_{i} / r\right)^{0.2}\right)$. The computation was then performed using Sobolev's large velocity gradient approximation (Sobolev 1960). The lower panel of Fig. 7 shows the results obtained from the model for two different gas temperatures $(4000 \mathrm{~K}$ and $10000 \mathrm{~K})$ and for a range of mass loss rates $\dot{M}_{\text {loss }}\left(10^{-6}-10^{-9} M_{\odot} \mathrm{yr}^{-1}\right)$ and envelope thickness $\left(1,2,5,10\right.$, and $\left.100 R_{*}\right)$.

In these models the line emission will be optically thick or thin depending on the assumed parameters. In any case, the locus of the results stretches from the central-upper part of the plot (intermediate values of $R$ ) towards the right-lower end of it (i.e. high $R$ values and decreasing $\operatorname{Pa} \beta$ flux). Albeit in a qualitative way, we can see that the sources displaying intermediatehigh $\mathrm{Pa} \beta / \mathrm{Br} \gamma$ ratios are located in the region compatible with the results of the wind model with a temperature of $4000 \mathrm{~K}$, in positions corresponding to mass loss rates of the order of $10^{-8}$ $10^{-9} M_{\odot} \mathrm{yr}^{-1}$. Actually, the same region of the $R-\log (\mathrm{Pa} \beta)$ plot can be covered also by the predictions of magnetospheric accretion models (see Muzerolle et al. 2001), although the expected gas temperatures are higher in this case ( $T \sim 6000-12000 \mathrm{~K})$.

In summary, our analysis of the $\mathrm{Pa} \beta / \mathrm{Br} \gamma$ ratio clearly shows the presence of at least two different $\mathrm{H}_{\mathrm{I}}$ emission modalities, one present in the objects showing low $R$ values and indicating optically thick lines, the other related to intermediate/high $R$ ratios compatible with optically thin emission. Any calibration of the empirical line- $L_{\text {acc }}$ relationships of $\mathrm{Br} \gamma$ and $\mathrm{Pa} \beta$ is clearly affected by the existence of these different emission modalities as shown by the $\mathrm{Br} \gamma-\mathrm{Pa} \beta$ comparison in Fig. 4, consequently it is important to understand their origin.

Observations of low $R$ values compatible with thermalised lines from small regions with gas at quite low temperatures have been reported also by Gatti et al. (2006) for many objects of their Ophiuchus sample, which is composed of low mass stars and brown dwarfs (BDs).

In this respect, it is interesting to compare our Fig. 7 with the analogous Fig. 7 of Gatti et al. (2006). We note that for the Cha sources the intrinsic $\mathrm{Pa} \beta$ flux of low-ratio objects appears to be higher $\left(10^{-14}-10^{-13} \mathrm{erg} \mathrm{s}^{-1} \mathrm{~cm}^{-2}\right)$ than in Ophiuchus BDs $\left(10^{-15}-10^{-14} \mathrm{erg} \mathrm{s}^{-1} \mathrm{~cm}^{-2}\right)$. Figure 7 of Gatti et al. (2006) also includes the results found by Muzerolle et al. (1998a) for the T Tauri stars in Taurus-Auriga, which we report in Fig. 7 as well. Remarkably, all sources of the Taurus-Auriga sample display high $\mathrm{Pa} \beta / \mathrm{Br} \gamma$ ratios $(R \gtrsim 3)$. Their position on the diagram is similar to the one of the Cha sources displaying intermediate-high values of $R$, i.e. these objects seem to present the same modality for the $\mathrm{H}_{\mathrm{I}}$ emission. Consistently with Fig. 7, Bary et al. (2008), who have extensively analysed the Brackett 
and Paschen decrements measured in the Taurus-Auriga CTTSs, conclude for example that $\mathrm{H}_{\mathrm{I}}$ emission is compatible with optically thin case-B recombination in a gas at very low temperature $(T \sim 2000 \mathrm{~K})$ and with electron densities of the order of $10^{10} \mathrm{~cm}^{-3}$.

We find that the majority of Taurus objects display fluxes of the order of $10^{-14}-10^{-13} \mathrm{erg} \mathrm{s}^{-1} \mathrm{~cm}^{-2}$, like most of our Cha sources. We also observe a few Cha objects that display $\mathrm{Pa} \beta$ fluxes on average higher $\left(\sim 10^{-13}-10^{-12} \mathrm{erg} \mathrm{s}^{-1} \mathrm{~cm}^{-2}\right.$, ten objects in the whole sample). However, there is no clear separation in terms of mass accretion rates between these objects and the others (see for example the comparison with Taurus sources in Fig. 8, which show $\dot{M}_{\text {acc }}$ values that uniformly span three orders of magnitude). Because we expect that the accretion luminosity is proportional to $\mathrm{Pa} \beta$, this result for $\dot{M}_{\text {acc }}$ must be caused by the mass of the objects. Indeed, we note that most of the Cha sources with higher $\mathrm{Pa} \beta$ fluxes actually have stellar masses $\left(\sim 1-2 M_{\odot}\right)$ larger than those typical of the Taurus sample (around $0.4 M_{\odot}$ ) (see e.g. Gatti et al. 2006).

In general, the higher $\mathrm{Pa} \beta$ fluxes in Cha could result if the emitting surface is larger (in the optically thick LTE case), or if the temperature is lower (in case B scenario), or if the $\dot{M}_{\text {loss }}$ is larger (for an LTE wind) in Cha than in most Taurus CTTSs or Ophiuchus BDs.

Regarding the origin of the $\mathrm{H}_{\mathrm{I}}$ emission in sources showing low $R$ values, Gatti et al. (2006) tentatively interpret this result in terms of optically thick line emission originating in the accretion spots on the protostellar photosphere: after the mass accretion rate has decreased below a given threshold value, the NIR H I optically thick emission component coming from the accretion spots would progressively become dominant with respect to the emission originating in the accretion columns. This interpretation appears problematic in our case, considered the emitting region sizes we derive from our observations (the reader is warned that the three blackbody emission curves in Fig. 7 of Gatti et al. 2006, refer, respectively, to emitting areas ten times smaller than ours), which would correspond to areas larger than the typical stellar disc. In any case, in this scenario we would expect a correlation between the observed low $R$ ratios and the mass accretion rate $\dot{M}_{\text {acc }}$.

To investigate this aspect, we analyse the ratio $\mathrm{Pa} \beta / \mathrm{Br} \gamma$ as a function of $\dot{M}_{\text {acc }}$ in Fig. 8. This can be compared to Fig. 5 of Gatti et al. (2006), which shows results for Ophiuchus BDs and for Taurus-Auriga CTTSs (Muzerolle et al. 1998a, also reported in our figure). In our sample, low $R$ values do not seem to be linked to a particular range of mass accretion rates, because they are observed in sources spanning a wide range of $\dot{M}_{\text {acc }}$ (3 dex), with no evident correlation between the two quantities. Conversely, there is a slight indication in our sample that the highest ratios $(R>4)$ are observed only in objects characterised by the lowest values of $\dot{M}_{\text {acc }}$, whereas high ratios are associated also with quite high mass accretion rates $\left(\sim 10^{-8}-10^{-6} M_{\odot} \mathrm{yr}^{-1}\right)$ in the Taurus CTTSs of Muzerolle et al. (1998a). To understand whether low $R$ sources possess any other common properties, we also looked for correlations between $R$ and other stellar properties. However, we find that $R$ does not clearly correlate with either the spectral index $\alpha_{2-24}, L_{*}, M_{*}$, or $T_{\text {eff }}$.

A viable scenario to explain the observed low ratios $R$ is the presence of an outflow, possibly associated with geometrical effects. For instance, the high-density regions at the base of a collimated jet seen almost face-on could likely be characterised by compact optically thick emission, where only the sections far from the star at lower temperature are observed. Alternatively, the $\mathrm{H}_{\mathrm{I}}$ emission might arise in the inner region of an optically

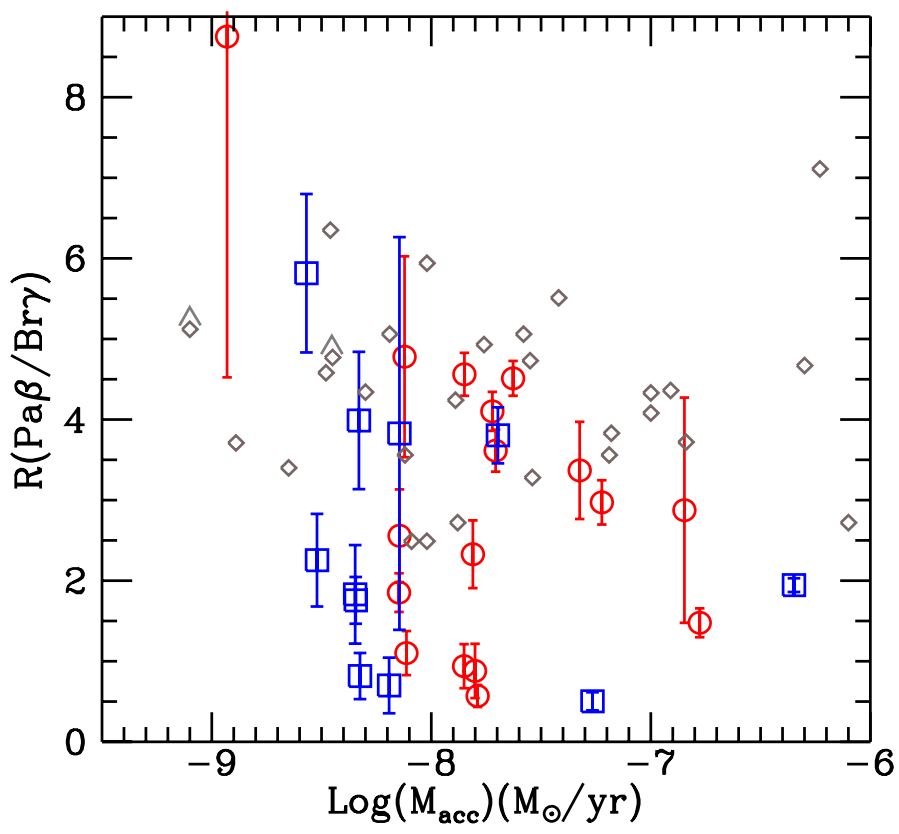

Fig. 8. $\dot{M}_{\text {acc }}$ as a function of the $\mathrm{Pa} \beta / \mathrm{Br} \gamma$ ratio. Red circles and blue squares refer to Cha I and Cha II sources, respectively. Measurements relative to Taurus CTTSs by Muzerolle et al. (1998a) are reported for comparison (grey diamonds).

thick gaseous disc. However, appropriate models for such a scenario have not been developed yet, because only the optically thin emission from the dust-free gaseous discs of relatively massive Herbig stars have been considered so far (e.g. Muzerolle et al. 2004).

\section{Conclusions}

As part of the POISSON project, we have presented the results of low-resolution spectroscopic optical-infrared observations $(0.6-2.4 \mu \mathrm{m})$ of 47 YSOs (mostly Class II objects) in the Chamaeleon I and II star-forming regions. The spectra, acquired with EFOSC2 and SofI at ESO/NTT, show many emission features commonly observed in young objects, in particular $\mathrm{H}_{\mathrm{I}}$ lines, $\mathrm{Ca}$ II, and [OI] emission. Taking advantage of the wide range of wavelengths covered by our data, we have considered five different accretion tracers ( $\mathrm{Br} \gamma, \mathrm{Pa} \beta, \mathrm{H} \alpha, \mathrm{Ca}$ II $\lambda 8542$, [O I] $\lambda 6300)$ to measure the accretion luminosity $L_{\text {acc }}$, making use of existing empirical relationships between emission line luminosity and $L_{\text {acc }}$. Our analysis primarily focussed on comparing $L_{\text {acc }}$ determinations from the five tracers, thereby allowing us to discuss the reliability and consistency of the different empirical relationships considered. From the derived accretion luminosities we have finally computed the mass accretion rate of the objects.

The main conclusions of our work can be summarised as follows:

- The different tracers provide $L_{\text {acc }}$ values characterised by different scatters when plotted as a function of $L_{*}$. The $\operatorname{Br} \gamma$ relationship (Calvet et al. 2004) appears to be the most reliable, because it gives the minimum dispersion of $L_{\text {acc }}$ throughout the range of $L_{*}$, whereas the other tracers, especially $\mathrm{H} \alpha$, provide much more scattered $L_{\text {acc }}$ results that are not expected for the homogeneous sample of targets observed. We therefore adopted the accretion luminosities computed from $\mathrm{Br} \gamma$. 
- The direct comparison between $L_{\mathrm{acc}}(\mathrm{Br} \gamma)$ and the accretion luminosity obtained from the other four tracers shows that the empirical relationships provide mean accretion luminosities that are systematically different in given ranges of $L_{*}$. These discrepancies as well as the large scatters may probably be ascribed to different excitation mechanisms (and/or emitting regions) contributing to the line, which may vary between our sample and those where the relationships were calibrated, which are basically composed of Taurus objects. Our analysis shows that the relationships for $\mathrm{H} \alpha$, [O I $]$, and $\mathrm{Ca}$ II are most subjected to these biases when applied to typical YSO samples.

- The derived accretion luminosities are in the range 0.1$1 L_{*}$ for all sources. The inferred mass accretion rates span from $10^{-7}$ to $10^{-9} M_{\odot} \mathrm{yr}^{-1}$, in the range of values commonly observed in Class IIs. The $\dot{M}_{\text {acc }}$ values computed in Cha I are roughly proportional to $M_{*}^{2}$, in agreement with the results found in other low-mass star-forming regions. Besides this dependence, we obtained indications of a slightly lower mean $\dot{M}_{\text {acc }}$ (for a given $M_{*}$ ) in Cha II than in Cha I. This might reflect the different age of the two associations, thus suggesting a decrease of the accretion rate with time.

- We found that for $L_{\mathrm{acc}}(\mathrm{Br} \gamma)$ and $L_{\mathrm{acc}}(\mathrm{Pa} \beta)$ the observed discrepancies can be actually related to different intrinsic $\mathrm{Pa} \beta / \mathrm{Br} \gamma$ ratios, which point to the existence of two different H I emission modalities.

The first one seems to agree generally with predictions of both wind and accretion models. Applying a simple spherical wind model, we obtained indications of gas temperatures around $4000 \mathrm{~K}$ and $\dot{M}_{\text {loss }}$ of $10^{-8}-10^{-9} M_{\odot} \mathrm{yr}^{-1}$.

The second one, associated with particularly low $\mathrm{Pa} \beta / \mathrm{Br} \gamma$ values $(R<2.5)$, indicates optically thick emission from regions with a size of the order of $10^{22} \mathrm{~cm}^{-3}$ (i.e. larger than the typical stellar surface area of an YSO) and low gas temperatures $T \lesssim 4000 \mathrm{~K}$. Possible scenarios include emission at the base of a jet, from an inner gaseous disc, or from accretion-heated spots on the stellar surface (at least in the case of smallest emission regions). Additional investigations are definitively required to ascertain the nature of the two different emission mechanisms observed.

Acknowledgements. The authors are grateful to K. L. Luhman for providing mass estimates for the Cha I objects. Alessio Caratti o Garatti acknowledges support from the Science Foundation of Ireland, grant 07/RFP/PHYF790, and the European Commision, grant ERG249157.

\section{Appendix: Empirical line-accretion relationships}

We report here the empirical line luminosity- $L_{\text {acc }}$ relationships for the tracers considered in our analysis, namely [O $\mathrm{I}] \lambda 6300$, Ca II 18542 , and the $\mathrm{H}_{\mathrm{I}}$ lines $\mathrm{H} \alpha, \mathrm{Pa} \beta$, and $\mathrm{Br} \gamma$, providing a short overview of the procedures used to derive the formulae. More details can be retrieved in the articles where the single relationships are provided.

In general, the reliability of a given relationship will depend on how accurately the accretion luminosity has been determined as well as on the range of stellar masses investigated. $\mathrm{UV} /$ blue-band excess measurements are the best direct accretion diagnostics (e.g. Hartmann et al. 1998; Gullbring et al. 1998, 2000; Herczeg \& Hillenbrand 2008), although their use is often limited owing to high extinctions typical of star-forming clouds. $L_{\text {acc }}$ values based on veiling estimates in other bands usually suffer from larger uncertainties (e.g. Herczeg \& Hillenbrand 2008). In this respect we note that the five relations considered are all initially based on observations of young sources of the Taurus-Auriga complex, for which these UV/blue-band measurements are available.

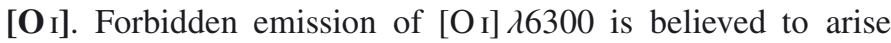
in winds/jets powered by accreting sources (e.g. Cabrit et al. 1990; Hamann et al. 1994; Hartigan et al. 1995), so that it is expected to correlate with $L_{\text {acc }}$, although in an indirect way. A relation between [O I] $\lambda 6300$ luminosity and accretion luminosity has been derived by Herczeg \& Hillenbrand (2008) from observations of a sample of young low-mass stars and brown dwarfs in Taurus, integrating their data with the results of spectroscopic measurements of CTTSs in the same region by Hartigan \& Kenyon (2003), to cover a range of stellar masses 0.05-1 $M_{\odot}$. Herczeg \& Hillenbrand (2008) derive accretion luminosities from direct measurement of the blue continuum excess, whereas Hartigan \& Kenyon (2003) provide $L_{\text {acc }}$ based on veiling in the $R$ band,

$\log L_{\mathrm{acc}} / L_{\odot}=0.96 \cdot \log L_{[\mathrm{O}] \mathrm{I}} / L_{\odot}+2.80$.

Ca II. The Ca II infrared triplet $(\lambda 8498, \lambda 8542, \lambda 8662)$ is believed to originate from gas in the magnetospheric accretion region, because the flux, in particular that of the $\lambda 8542$ line, appears to correlate strongly with the accretion rate (Muzerolle et al. 1998a). However, these lines have been detected also in jets (Nisini et al. 2005b; Podio et al. 2006), which indicates that Ca II might be (at least in part) an indirect tracer. We adopt the $L_{\text {acc}}-\mathrm{Ca}$ II $\lambda 8542$ relationship computed by Dahm (2008) on the basis of the accretion luminosities of 14 Taurus-Auriga sources, established either through $U$-band photometry or blue excess measurement, mostly taken from Gullbring et al. (1998) and (Muzerolle et al. 1998a), which have masses $\lesssim 1 M_{\odot}$. Dahm (2008) then applied this relationship to his IC348 cluster sample, which displays a range of masses $\left(0.5-2.0 M_{\odot}\right)$ similar to that of our sample:

$\log L_{\text {acc }} / L_{\odot}=1.02 \cdot \log L_{\mathrm{Ca} \text { II }} / L_{\odot}+2.50$.

$\mathbf{H} \boldsymbol{\alpha} . \mathrm{H} \alpha$ equivalent width has been traditionally used in the past to distinguish between the so-called classical and weak T Tauri stars, that is, between actively accreting $\mathrm{T}$ Tauri stars and those that are relatively devoid of circumstellar matter. The $\mathrm{H} \alpha$ velocity width at $10 \%$ of the peak has been found to be a good diagnostic of the accretion (e.g. White \& Basri 2003; Natta et al. 2004). As for the flux of the line, the empirical correlation between accretion and $\mathrm{H} \alpha$ has been recently revised by Fang et al. (2009), who have combined the available data from Gullbring et al. (1998); Dahm (2008); Herczeg \& Hillenbrand (2008) to derive the following relationship:

$\log L_{\mathrm{acc}} / L_{\odot}=1.25 \cdot \log L_{\mathrm{H} \alpha} / L_{\odot}+2.27$.

$\operatorname{Pa} \beta$ and $\operatorname{Br} \gamma$. The correlation of the $\operatorname{Pa} \beta$ and $\operatorname{Br} \gamma$ lines with the accretion luminosity has been investigated in several works during the last years (see e.g. Muzerolle et al. 1998b; Calvet et al. 2000, 2004; Natta et al. 2004; Nisini et al. 2005a). These correlations are particularly important because they provide a straightforward method to compute $L_{\text {acc }}$ for young embedded sources that are observable only at infrared wavelengths, and for which it is consequently impossible to measure accretion directly through the detection of the UV-optical veiling. $\mathrm{H}_{\mathrm{I}}$ infrared emission has therefore been extensively used as tracer to derive the accretion rate in young objects (see e.g. Natta et al. 2006; Garcia Lopez et al. 2006; Antoniucci et al. 2008) For our sample we adopt the 
empirical laws obtained by Calvet et al. (2000, 2004), based on accretion luminosities derived from the UV excess observed in a sample of Taurus and Orion objects. This extends the relations found by Muzerolle et al. (1998a) for low-mass CTTSs to more massive pre-main sequence objects (up to $4 M_{\odot}$ ),

$\log L_{\text {acc }} / L_{\odot}=1.03 \cdot \log L_{\mathrm{Pa} \beta} / L_{\odot}+2.8$

$\log L_{\mathrm{acc}} / L_{\odot}=0.9 \cdot \log L_{\mathrm{Br} \gamma} / L_{\odot}+2.9$.

\section{References}

Alcalá, J. M., Spezzi, L., Chapman, N., et al. 2008, ApJ, 676, 427

Allers, K. N., Kessler-Silacci, J. E., Cieza, L. A., \& Jaffe, D. T. 2006, ApJ, 644, 364

Alves de Oliveira, C., \& Casali, M. 2008, A\&A, 485, 155

Andre, P., Ward-Thompson, D., \& Barsony, M. 1993, ApJ, 406, 122

Antoniucci, S., Nisini, B., Giannini, T., \& Lorenzetti, D. 2008, A\&A, 479, 503

Baraffe, I., Chabrier, G., Allard, F., \& Hauschildt, P. H. 1998, A\&A, 337, 403

Bary, J. S., Matt, S. P., Skrutskie, M. F., et al. 2008, ApJ, 687, 376

Beck, T. L. 2007, AJ, 133, 1673

Beck, T. L., McGregor, P. J., Takami, M., \& Pyo, T.-S. 2008, ApJ, 676, 472

Beck, T. L., Bary, J. S., \& McGregor, P. J. 2010, ApJ, 722, 1360

Cabrit, S., Edwards, S., Strom, S. E., \& Strom, K. M. 1990, ApJ, 354, 687

Calvet, N., Hartmann, L., \& Hewett, R. 1992, ApJ, 386, 229

Calvet, N., Hartmann, L., \& Strom, S. E. 2000, Protostars and Planets IV, 377

Calvet, N., Muzerolle, J., Briceño, C., et al. 2004, AJ, 128, 1294

Cardelli, J. A., Clayton, G. C., \& Mathis, J. S. 1989, ApJ, 345, 245

Connelley, M. S., \& Greene, T. P. 2010, AJ, 140, 1214

Dahm, S. E. 2008, AJ, 136, 521

Damjanov, I., Jayawardhana, R., Scholz, A., et al. 2007, ApJ, 670, 1337

D’Antona, F., \& Mazzitelli, I. 1997, Mem. Soc. Astron. Ital., 68, 807

D'Odorico, S. 1988, The Messenger, 52, 51

Edwards, S., Fischer, W., Hillenbrand, L., \& Kwan, J. 2006, ApJ, 646, 319

Enoch, M. L., Evans, N. J., Sargent, A. I., \& Glenn, J. 2009, ApJ, 692, 973

Evans, N. J., Dunham, M. M., Jørgensen, J. K., et al. 2009, ApJS, 181, 321

Fang, M., van Boekel, R., Wang, W., et al. 2009, A\&A, 504, 461

Folha, D. F. M., \& Emerson, J. P. 2001, A\&A, 365, 90

Garcia Lopez, R., Natta, A., Testi, L., \& Habart, E. 2006, A\&A, 459, 837

García López, R., Nisini, B., Antoniucci, S., et al. 2011, A\&A, in press

Gatti, T., Testi, L., Natta, A., Randich, S., \& Muzerolle, J. 2006, A\&A, 460, 547

Gómez, M., \& Persi, P. 2002, A\&A, 389, 494

Greene, T. P., \& Lada, C. J. 1996, AJ, 112, 2184

Gullbring, E., Hartmann, L., Briceno, C., \& Calvet, N. 1998, ApJ, 492, 323

Gullbring, E., Calvet, N., Muzerolle, J., \& Hartmann, L. 2000, ApJ, 544, 927

Hamann, F., \& Persson, S. E. 1992, ApJS, 82, 247

Hamann, F., Depoy, D. L., Johansson, S., \& Elias, J. 1994, ApJ, 422, 626

Hartigan, P., \& Kenyon, S. J. 2003, ApJ, 583, 334

Hartigan, P., Edwards, S., \& Ghandour, L. 1995, ApJ, 452, 736

Hartmann, L., Calvet, N., Gullbring, E., \& D’Alessio, P. 1998, ApJ, 495, 385

Herczeg, G. J., \& Hillenbrand, L. A. 2008, ApJ, 681, 594

Hirth, G. A., Mundt, R., \& Solf, J. 1997, A\&AS, 126, 437
Hummer, D. G., \& Storey, P. J. 1987, MNRAS, 224, 801

Lada, C. J., \& Wilking, B. A. 1984, ApJ, 287, 610

Luhman, K. L. 2004, ApJ, 602, 816

Luhman, K. L. 2007, ApJS, 173, 104

Luhman, K. L., \& Muench, A. A. 2008, ApJ, 684, 654

Luhman, K. L., Lada, C. J., Hartmann, L., et al. 2005, ApJ, 631, L69

Luhman, K. L., Allen, L. E., Allen, P. R., et al. 2008, ApJ, 675, 1375

Melnikov, S. Y., Eislöffel, J., Bacciotti, F., Woitas, J., \& Ray, T. P. 2009, A\&A, 506, 763

Mendigutía, I., Eiroa, C., Montesinos, B., et al. 2011, A\&A, 529, A34

Meyer, M. R., Calvet, N., \& Hillenbrand, L. A. 1997, AJ, 114, 288

Mizuno, A., Hayakawa, T., Tachihara, K., et al. 1999, PASJ, 51, 859

Mohanty, S., Jayawardhana, R., \& Basri, G. 2005, ApJ, 626, 498

Morwood, A. 1997, The Messenger, 88, 11

Muzerolle, J., Hartmann, L., \& Calvet, N. 1998a, AJ, 116, 2965

Muzerolle, J., Hartmann, L., \& Calvet, N. 1998b, AJ, 116, 455

Muzerolle, J., Calvet, N., \& Hartmann, L. 2001, ApJ, 550, 944

Muzerolle, J., D’Alessio, P., Calvet, N., \& Hartmann, L. 2004, ApJ, 617, 406

Muzerolle, J., Luhman, K. L., Briceño, C., Hartmann, L., \& Calvet, N. 2005, ApJ, 625, 906

Najita, J., Carr, J. S., \& Mathieu, R. D. 2003, ApJ, 589, 931

Natta, A., Testi, L., Muzerolle, J., et al. 2004, A\&A, 424, 603

Natta, A., Testi, L., \& Randich, S. 2006, A\&A, 452, 245

Nisini, B., Milillo, A., Saraceno, P., \& Vitali, F. 1995, A\&A, 302, 169

Nisini, B., Antoniucci, S., Giannini, T., \& Lorenzetti, D. 2005a, A\&A, 429, 543

Nisini, B., Bacciotti, F., Giannini, T., et al. 2005b, A\&A, 441, 159

Palla, F., \& Stahler, S. W. 1999, ApJ, 525, 772

Podio, L., Bacciotti, F., Nisini, B., et al. 2006, A\&A, 456, 189

Porras, A., Jørgensen, J. K., Allen, L. E., et al. 2007, ApJ, 656, 493

Ray, T., Dougados, C., Bacciotti, F., Eislöffel, J., \& Chrysostomou, A. 2007, Protostars and Planets V, 231

Reipurth, B., \& Bally, J. 2001, ARA\&A, 39, 403

Reipurth, B., Pedrosa, A., \& Lago, M. T. V. T. 1996, A\&AS, 120, 229

Rieke, G. H., \& Lebofsky, M. J. 1985, ApJ, 288, 618

Rigliaco, E., Natta, A., Randich, S., Testi, L., \& Biazzo, K. 2011, A\&A, 525, A47

Scholz, A., Jayawardhana, R., \& Brandeker, A. 2005, ApJ, 629, L41

Schwartz, R. D., Wilking, B. A., \& Giulbudagian, A. L. 1991, ApJ, 370, 263

Skrutskie, M. F., Cutri, R. M., Stiening, R., et al. 2006, AJ, 131, 1163

Sobolev, V. V. 1960, Moving envelopes of stars (Cambridge: Harvard University Press)

Spezzi, L., Alcalá, J. M., Covino, E., et al. 2008, ApJ, 680, 1295

Stelzer, B., Scholz, A., \& Jayawardhana, R. 2007, ApJ, 671, 842

Tarenghi, M., \& Wilson, R. N. 1989, in SPIE Conf. Ser. 1114, ed. F. J. Roddier, 302

Vrba, F. J., \& Rydgren, A. E. 1984, ApJ, 283, 123

White, R. J., \& Basri, G. 2003, ApJ, 582, 1109

White, R. J., \& Hillenbrand, L. A. 2004, ApJ, 616, 998

White, R. J., Greene, T. P., Doppmann, G. W., Covey, K. R., \& Hillenbrand, L. A. 2007, in Protostars and Planets V, ed. B. Reipurth, D. Jewitt, \& K. Keil, 117

Whittet, D. C. B., Gerakines, P. A., Carkner, A. L., et al. 1994, MNRAS, 268, 1

Whittet, D. C. B., Prusti, T., Franco, G. A. P., et al. 1997, A\&A, 327, 1194

Young, K. E., Harvey, P. M., Brooke, T. Y., et al. 2005, ApJ, 628, 283 